\title{
Inductive Invariant Checking with Partial Negative Application Conditions
}

Johannes Dyck, Holger Giese

Technische Berichte Nr. 98

des Hasso-Plattner-Instituts für

Softwaresystemtechnik

an der Universität Potsdam

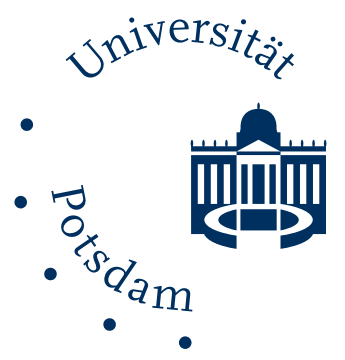



Technische Berichte des Hasso-Plattner-Instituts für Softwaresystemtechnik an der Universität Potsdam 

Technische Berichte des Hasso-Plattner-Instituts für Softwaresystemtechnik an der Universität Potsdam | 98

Johannes Dyck | Holger Giese

Inductive Invariant Checking with Partial Negative Application Conditions 


\section{Bibliografische Information der Deutschen Nationalbibliothek}

Die Deutsche Nationalbibliothek verzeichnet diese Publikation in der Deutschen Nationalbibliografie; detaillierte bibliografische Daten sind im Internet über http:/ /dnb.dnb.de/ abrufbar.

Universitätsverlag Potsdam 2015

http:/ / verlag.ub.uni-potsdam.de/

Am Neuen Palais 10, 14469 Potsdam

Tel.: +49 (0)331 9772533 / Fax: 2292

E-Mail: verlag@uni-potsdam.de

Die Schriftenreihe Technische Berichte des Hasso-Plattner-Instituts für

Softwaresystemtechnik an der Universität Potsdam wird herausgegeben von den Professoren des Hasso-Plattner-Instituts für Softwaresystemtechnik an der Universität Potsdam.

ISSN (print) 1613-5652

ISSN (online) 2191-1665

Das Manuskript ist urheberrechtlich geschützt.

Online veröffentlicht auf dem Publikationsserver der Universität Potsdam

URN urn:nbn:de:kobv:517-opus4-77748

http:/ / nbn-resolving.de/urn:nbn:de:kobv:517-opus4-77748

Zugleich gedruckt erschienen im Universitätsverlag Potsdam:

ISBN 978-3-86956-333-6 
Graph transformation systems are a powerful formal model to capture model transformations or systems with infinite state space, among others. However, this expressive power comes at the cost of rather limited automated analysis capabilities. The general case of unbounded many initial graphs or infinite state spaces is only supported by approaches with rather limited scalability or expressiveness. In this paper we improve an existing approach for the automated verification of inductive invariants for graph transformation systems. By employing partial negative application conditions to represent and check many alternative conditions in a more compact manner, we can check examples with rules and constraints of substantially higher complexity. We also substantially extend the expressive power by supporting more complex negative application conditions and provide higher accuracy by employing advanced implication checks. The improvements are evaluated and compared with another applicable tool by considering three case studies. 



\section{Introduction}

Graph transformation systems are a powerful formal model to capture model transformations, systems with reconfiguration, or systems with infinite state space, among others. However, the expressive power of graph transformation systems comes at the cost of rather limited automated analysis capabilities.

While for graph transformation systems with finite state space of moderate size certain model checkers can be used (e.g., [9, 16]), in the general case of unbounded many initial graphs or an infinite state space only support by techniques with rather limited scalability or expressiveness exists.

There is a number of automated approaches that can handle infinite state spaces by means of abstraction [5, 17, 14, 13], but they are considerably limited in expressive power as they only support limited forms of negative application conditions at most. Tools only targeting invariants [4, 2] also only support limited forms of negative application conditions at most; in some cases additional limitations concerning the graphs of the state space apply (cf. [4]). On the other hand the SeekSat/ProCon tool [15, 11] is able to prove correctness of graph programs with respect to pre- and postconditions specified as nested graph constraints without such limitations, but requires potentially expensive computations.

In this paper we present improvements of our existing approach introduced in [2] for the automated verification of inductive invariants for graph transformation systems. Inductive invariants are properties whose validity before the application of a graph rule implies their validity thereafter. Our general approach involves the construction of a violation of the invariant after application of a graph rule, represented in a symbolic way (target pattern), followed by calculation of the symbolic state before rule application (source pattern). If a violation can then be found in all such source patterns, the rule does not violate the inductive invariant; otherwise, it does and the construction yields a witness. Since inductive invariants are checked with respect to the capability of individual rules to violate or preserve them, this technique avoids the computationally expensive computation of state spaces and can even handle infinite systems.

By employing partial negative application conditions to represent and check many alternative conditions in a more compact manner, our approach is now able to check examples with rules and constraints of substantially higher complexity. Our improvements also provide higher accuracy by employing advanced implication checks and extend expressive power by supporting more complex negative application conditions. While not as expressive as the general concept of nested graph conditions [11], there is a significant number of examples [2, 10, 3] for which the supported level of expressive power is sufficient. Of those, we employ three case studies concerned with car platooning and model transformations to evaluate our 
improvements and to compare them with the SeekSat/ProCon tool, demonstrating that our approach shows better scalability for certain cases.

The report is organized as follows: The formal foundations are introduced in Section 2. Our restrictions and important constructions in our algorithms are explained in Section 3 . Section 4 presents the employed inductive invariant checking scheme with its formal justification. Section 5 presents our evaluation, with Section 6 then discussing related work. Finally, Section 7 provides a summary and outlook on possible future work. Additional prerequisites concerning the formal model can be found in Appendix $\mathrm{A}$ and $\mathrm{B}$; omitted proofs can be found in the respective sources. 


\section{Foundations}

This section shortly describes foundations of graph transformation systems we use in our verification approach. For additional definitions, we refer to Appendix A.

The formalism used herein (cf. [7]) considers a graph $G=(V, E, s, t)$ to consist of sets of nodes, edges and source and target functions $s, t: E \rightarrow V$. A graph morphism $f: G_{1} \rightarrow G_{2}$ consists of two functions mapping nodes and edges, respectively, that preserve source and target functions. In this paper we put special emphasis on injective morphisms (or monomorphisms), denoted $f: G_{1} \hookrightarrow G_{2}$, and consider typed graphs, i.e. graphs typed over a type graph $T G$ by a typing morphism type: $G \rightarrow T G$ and typed graph morphisms that preserve the typing morphism. We also adopt the concept of partial monomorphisms:

Definition 1 (partial monomorphism ([15], adjusted)). A partial monomorphism $p: A \sqcup B$ is a 2-tuple $p=\langle a, b\rangle$ of monomorphisms $a, b$ with $\operatorname{dom}(a)=\operatorname{dom}(b), \operatorname{dom}(p)=$ $\operatorname{codom}(a)$, and $\operatorname{codom}(p)=\operatorname{codom}(b)$. The interface of $p$ refers to the common domain of $a$ and $b$, i.e., iface $(p)=\operatorname{dom}(a)=\operatorname{dom}(b)$. A partial monomorphism $p=\langle a, b\rangle$ is said to be a total monomorphism $b$, if $a$ is an isomorphism, i.e. a bijective morphism.

Thus, the partial monomorphism $p: A \sqcup B$ describes an inclusion of a subgraph $A^{\prime}$ of $A$ in $B$. With partial monomorphisms we can define partial application conditions, which, similar to nested application conditions [11], describe conditions on mor-

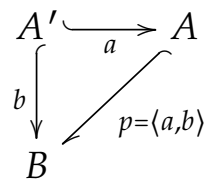
phisms. Graph constraints, on the other hand, describe conditions on graphs.

Definition 2 (partial application condition ([8], extended to partial morphisms)). $A$ partial application condition is inductively defined as follows:

1. For every graph $P$, true is a partial application condition over $P$.

2. For every partial monomorphism $a: P \sqcup C$ with $a=\langle p, c\rangle$ and monomorphisms $p: P^{\prime} \rightarrow P$ and $c: P^{\prime} \rightarrow C$ and every partial application condition ac over $C, \exists(a, a c)$ is an application condition over $P$.

3. For partial application conditions $a c$, ac $c_{i}$ over $P$ with $i \in I$ (for all index sets $I$ ), $\neg$ ac and $\bigwedge_{i \in I} a c_{i}$ are partial application conditions over $P$.
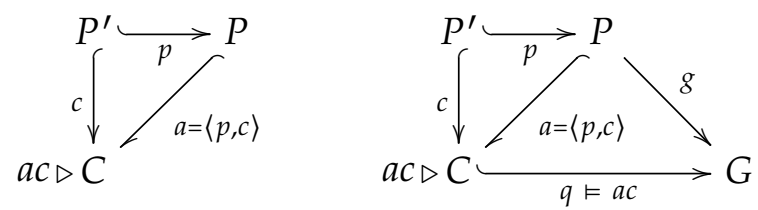
Satisfiability of partial application conditions is inductively defined as follows:

1. Every morphism satisfies true.

2. A morphism $g: P \rightarrow G$ satisfies $\exists(a, a c)$ over $P$ with $a: P \sqcup C$ with $a=\langle p, c\rangle$ if there exists an injective $q: C \rightarrow G$ such that $q \circ C=g \circ p$ and $q$ satisfies ac.

3. A morphism $g: P \rightarrow G$ satisfies $\neg$ ac over $P$ if $g$ does not satisfy ac and $g$ satisfies $\bigwedge_{i \in I} a c_{i}$ over $P$ if $g$ satisfies each $a c_{i}(i \in I)$.

We write $g \vDash$ ac to denote that the morphism $g$ satisfies ac.

Two application conditions $a c$ and $a c^{\prime}$ are equivalent, denoted by $a c \equiv a c^{\prime}$, if for all morphisms $g: P \rightarrow G, g \vDash a c$ if and only if $g \vDash a c^{\prime}$.

If all morphisms involved in a partial application condition are total morphisms we say that it is a total application condition.

$\exists p$ abbreviates $\exists(p$, true $) . \forall(p, a c)$ abbreviates $\neg \exists(p, \neg a c)$.

Definition 3 (graph constraint [11]). A graph constraint is an application condition over the empty graph $\varnothing$. A graph $G$ then satisfies such a condition if the initial morphism $i_{G}: \varnothing \hookrightarrow G$ satisfies the condition.

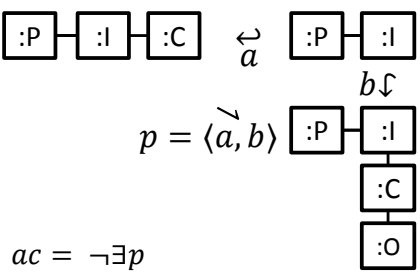

(a) Partial condition

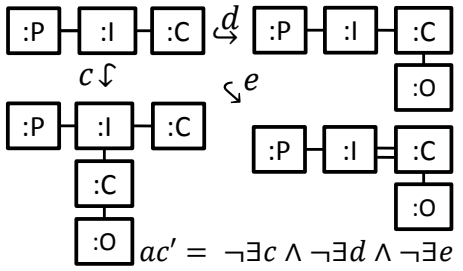

(b) Total condition

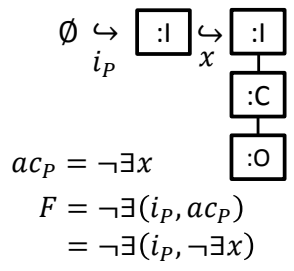

(c) Graph constraint

Figure 1: Partial and total conditions and graph constraint

Example 4. Figure 1 shows an example from a software refactoring context (cf. [3]) with node types $P, I, C, O$ standing for Package, Interface, Class, and Operation, respectively. Although equivalent, the partial condition ac in Figure $\mathbf{I}(a)$ is much more compact - and also less expensive in computation - when compared to the total condition ac' in Figure $\mathbf{1}(b)$ Both conditions describe the absence of an implementing class and contained operation for the interface. Further, Figure $1(c)$ shows a graph constraint $F$, which forbids the existence of an interface without an implementing class containing an operation.

Application conditions can also be used in graph rules, which are used to transform graphs. Finally, a graph transformation system consists of a number of rules and, in our case of typed graph transformation systems, of a type graph. 
Definition 5 (rules and transformations [8]). A plain rule $p=(L \hookleftarrow K \hookrightarrow R)$ consists of two injective morphisms $K \rightarrow L$ and $K \rightarrow R$. $L$ and $R$ are called left- and right-hand side of $p$, respectively. A rule $b=\left\langle p, a c_{L}, a c_{R}\right\rangle$ consists of a plain rule $p$ and $a$ left (right) application condition $a c_{L}\left(a c_{R}\right)$ over $L(R)$.

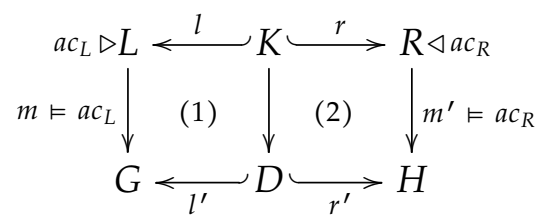

A direct transformation consists of two pushouts (1) and (2) such that $m \vDash a_{L}$ and $m^{\prime} \vDash a c_{R}$. We write $G \Rightarrow_{b, m, m^{\prime}} H$ and say that $m: L \rightarrow G$ is the match of $b$ in $G$ and $m^{\prime}: R \rightarrow H$ is the comatch of $b$ in $H$. We also write $G \Rightarrow_{b, m} H, G \Rightarrow_{m} H$ or $G \Rightarrow H$ to express that there exist $m^{\prime}, m$ or $b$ such that $G \Rightarrow_{b, m, m^{\prime}} H$.

We also introduce the concept of a reduced rule, which basically is a rule without certain elements irrelevant for a specific application via a match once the applicability for that match is ensured. By using reduced rules, we can reduce the effort necessary for verification, as will be shown later.

Definition 6 (reduced rule). Given a plain rule $b=\langle L \leftrightarrow K \hookrightarrow R\rangle$, we define a reduced rule of $b$ as a rule $b^{*}=\left\langle L^{*} \leftrightarrow K^{*} \hookrightarrow R^{*}\right\rangle$ with injective morphisms $r^{+}: R^{*} \hookrightarrow R, l^{+}: L^{*} \hookrightarrow L$ and $k^{+}: K^{*} \hookrightarrow K$ such that for all graphs $G, H$ and injective morphisms $m, m^{\prime}$ it holds that $G \Rightarrow_{b, m, m^{\prime}} H \Leftrightarrow G \Rightarrow_{b^{*}, m o l^{+}, m^{\prime} \circ r^{+}} H$.

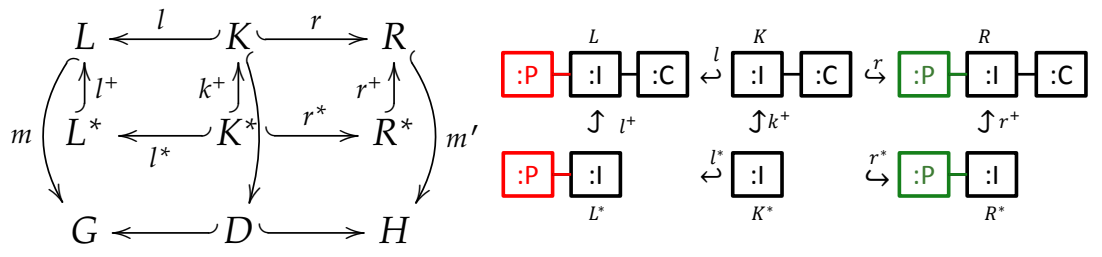

Example 7. The figure above shows a plain rule describing the replacement of a package containing an existing interface and class. In general, a corresponding reduced rule (also depicted) can be constructed by choosing $K^{*}$ as any subgraph of $K$ whose images under $l$ and $r$ include all nodes attached to edges to be deleted or created and then constructing $L^{*}$ and $R^{*}$ as the pushout complements of $\left\langle k^{+}, l\right\rangle$ and $\left\langle k^{+}, r\right\rangle$, respectively. 


\section{Restrictions, Constructions, and Implication}

With the foundations established, we will now introduce certain restrictions that apply to our specifications and the main constructions used by our algorithms.

The most important adjustments are concerned with the notion of rules and application conditions. Since most application conditions that will be encountered in this paper have the same structure, we define a special kind of negative application conditions without additional nesting. In comparison to our previous work [2], this is a significant difference in expressive power, as [2] allowed only negative application conditions with each having a node and an edge, at most.

Definition 8 (composed negative application condition). A composed negative application condition is an application condition of the form ac $=\Lambda_{i \in I} \neg \exists a_{i}$ for partial monomorphisms $a_{i}$ of a common domain. An individual condition $\neg \exists a_{i}$ is called negative application condition. A (composed) total negative application condition is a (composed) negative application condition including only total graph morphisms.

Our properties for verification are described by so-called forbidden patterns:

Definition 9 (pattern). A pattern is a graph constraint of the form $F=\exists\left(\varnothing \hookrightarrow P, a c_{P}\right)$, with $P$ being a graph and $a_{P}$ a composed total negative application condition over $P$. A composed forbidden pattern is a graph constraint of the form $\mathcal{F}=\bigwedge_{i \in I} \neg F_{i}$ for some index set $I$ and patterns $F_{i}$. Patterns $F_{i}$ occurring in a composed forbidden pattern are also called forbidden patterns.

We also allow graph transformation systems to be equipped with a special variant of composed forbidden pattern called composed guaranteed pattern. Such a pattern is a constraint whose validity is guaranteed by some external means or additional knowledge about the system under verification.

While our specification language concerning patterns and application conditions is more limited than the general concept of nested application conditions [11], the level of expressive power we support is sufficient to verify a number of case studies [2, 10, 3]. On the other hand, the following additional limitations in our approach (except for the second) do not result in a loss of expressive power [11, 7, 8]:

Morphisms in application conditions (Definition 2) must be injective.

Left application conditions (Definition 5) in rules are required to be composed total negative application conditions.

Right application conditions (Definition 5) in rules are required to be true.

Rule applicability (Definition 5) requires injective matches and comatches. 
To conclude the definitions used in our verification approach, we introduce our notion of inductive invariants for graph transformation systems. Informally, all rule applications should preserve the validity of a composed forbidden pattern $\mathcal{F}$. Since the system is assumed to prevent violations of a composed guaranteed pattern $\mathcal{G}$ by other means (e.g., a postprocessing step) or additional knowledge, rule applications leading to such a violation do not need to be considered.

Definition 10 (inductive invariant). Given a composed forbbidden pattern $\mathcal{F}$ and $a$ composed guaranteed pattern $\mathcal{G}$, a typed graph transformation system $G T S=(T G, B)$ is preserving $\mathcal{F}$ under $\mathcal{G}$ if, for each rule $b$ in $B$, it holds that

$$
\forall G, H\left(\left(G \Rightarrow_{b} H\right) \Longrightarrow((G \vDash \mathcal{F} \wedge G \vDash \mathcal{G}) \Rightarrow(H \vDash \mathcal{F} \vee H \not \mathcal{G}))\right)
$$

A composed forbidden pattern $\mathcal{F}$ preserved by GTS under $\mathcal{G}$ is an inductive invariant for GTS under $\mathcal{G}$.

\subsection{Constructions}

An important part of our algorithm is the transformation of application conditions over morphisms and rules. [8] presents a Shift-construction for a transformation of application conditions over morphisms into equivalent application conditions. For our restricted formal model, we use a marginally adjusted form of the Shiftconstruction. Its validity is proven in Lemma 36 in Appendix B.

Construction 11 (Shift-construction, adjusted from [8]). For each total application condition ac over a graph $P$ and for each morphism $b: P \rightarrow P^{\prime}$, Shift $(b, a c)$ transforms ac via $b$ into a total application condition over $P^{\prime}$ such that, for each morphism $n: P^{\prime} \leftrightarrow H$, it holds that $n \circ b \vDash a c \Leftrightarrow n \vDash \operatorname{Shift}(b, a c)$.

The Shift-construction is inductively defined as follows:

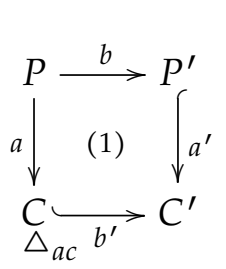

$$
\text { Shift }(b, \text { true })=\text { true. }
$$$$
\text { Shift }(b, \exists(a, a c))=\bigvee_{\left(a^{\prime}, b^{\prime}\right) \in \mathcal{F}} \exists\left(a^{\prime}, \text { Shift }\left(b^{\prime}, a c\right)\right) \text { if }
$$

$\mathcal{F}=\left\{\left(a^{\prime}, b^{\prime}\right) \mid\left(a^{\prime}, b^{\prime}\right)\right.$ are jointly surjective, $a^{\prime}, b^{\prime}$ are injective, and ( 1 ) commutes $\left.\left(b^{\prime} \circ a=a^{\prime} \circ b\right)\right\} \neq \varnothing$ and false, otherwise.

Shift $(b, \neg a c)=\neg$ Shift $(b, a c)$. $\operatorname{Shift}\left(b, \wedge_{i \in I} a c_{i}\right)=\bigwedge_{i \in I} \operatorname{Shift}\left(b, a c_{i}\right)$.

While this construction can be employed to equivalently transform total application conditions, the calculation of the respective morphism pairs is computationally expensive. To avoid executing that calculation, we construct partial application conditions instead and establish their equivalence to the result of the Shift-construction in the following construction and lemma. As before, proof of validity and a more detailed version can be found in Lemma 40 in Appendix B. 
Construction 12 (PShift-construction). For each total application condition ac over $P^{\prime}$ and for each morphism $p^{\prime}: P^{\prime} \hookrightarrow P$, PShift $\left(p^{\prime}, a c\right)$ transforms ac via $p^{\prime}$ into a partial application condition over $P$ such that, for each morphism $n: P \hookrightarrow H$, it holds that $n \circ p^{\prime} \vDash a c \Leftrightarrow n \vDash \operatorname{PShift}\left(p^{\prime}, a c\right)$.

The PShift-construction is defined as follows:

$$
\begin{array}{ll}
P^{\prime} \stackrel{p^{\prime}}{\longrightarrow} P & \text { PShift }\left(p^{\prime}, \text { true }\right)=\text { true. } \\
\left.\downarrow_{C}^{a} \operatorname{PShift}_{c=\left\langle p^{\prime}, a^{\prime}\right\rangle}, \exists(a, a c)\right)=\exists(c, a c) \text { with } c=\left\langle p^{\prime}, a\right\rangle . & \operatorname{PShift}\left(p^{\prime}, \neg a c\right)=\neg P \operatorname{PSh}\left(p^{\prime}, a c\right) . \\
\triangle_{a c} & \operatorname{PShift}\left(p^{\prime}, \bigwedge_{i \in I} a c_{i}\right)=\bigwedge_{i \in I} \operatorname{PShift}\left(p^{\prime}, a c_{i}\right) .
\end{array}
$$

Lemma 13 (equivalence of Shift and PShift). For each application condition ac over $P$ and each monomorphism $p^{\prime}: P \hookrightarrow P^{\prime}$, we have Shift $\left(p^{\prime}, a c\right) \equiv \operatorname{PShift}\left(p^{\prime}, a c\right)$.
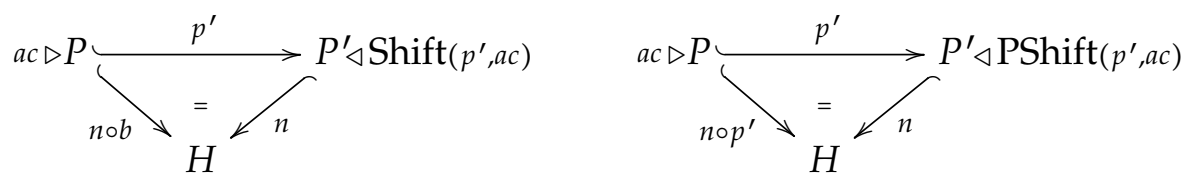

Proof. This follows directly from Lemma 36 . Lemma 40 and the respective constructions.

We also transform application conditions over rules using the L-construction found in [8]. Lemma 37 in Appendix B describes the formal basis for this construction.

Construction 14 (L-construction [8, 11]). For each rule $b=\langle L \hookleftarrow K \hookrightarrow R\rangle$ and each total application condition ac over $R, L(b, a c)$ transforms ac via $b$ into a total application condition over $L$ such that, for each direct transformation $G \Rightarrow_{b, m, m^{\prime}} H$, we have $m \vDash$ $L(b, a c) \Leftrightarrow m^{\prime} \vDash a c$.

The L-construction is inductively defined:

$$
\begin{aligned}
& L(b, \text { true })=\text { true } . \\
& L(b, \exists(a, a c))=\exists\left(a^{\prime}, L\left(b^{\prime}, a c\right)\right) \text { (with } b^{\prime}= \\
& \left\langle L^{\prime} \hookleftarrow K^{\prime} \leftrightarrow R^{\prime}\right\rangle \text { constructed via the pushouts (1) } \\
& \text { and (2)) if }\langle r, a\rangle \text { has a pushout complement (1) and } \\
& L\left(b^{\prime}, a c\right) \triangleright L^{\prime} \longleftarrow K_{l^{\prime}}^{\prime} \underbrace{\longrightarrow}_{r^{\prime}} R^{\prime} \triangleleft a c \\
& \text { false, otherwise. } \\
& L(b, \neg a c)=\neg L(b, a c) \text {. } \\
& L\left(b, \bigwedge_{i \in I} a c_{i}\right)=\bigwedge_{i \in I} L\left(b, a c_{i}\right) .
\end{aligned}
$$




\subsection{Implication}

One of the main requirements for our algorithm is the comparison of graph constraints or, more precisely, the notion of implication of patterns.

Definition 15 (implication of patterns). Let $C=\exists(\varnothing \hookrightarrow P, a c)$ and $C^{\prime}=\exists\left(\varnothing \hookrightarrow P^{\prime}, a c^{\prime}\right)$ with composed partial negative application conditions ac and $a c^{\prime}$ be two patterns. $C^{\prime}$ implies $C\left(C^{\prime} \vDash C\right)$, if the following condition holds:

$$
\forall G\left(G \vDash C^{\prime} \Rightarrow G \vDash C\right) .
$$

Since a pattern may be fulfilled by an infinite number of graphs, we cannot (in general) check the above condition for all such graphs. Instead, we establish a condition sufficient to imply implication when comparing patterns. Depending on whether the patterns' application conditions ac and $\mathrm{ac}^{\prime}$ are partial, total, or nonexistent (i.e. true), the procedure and its computational effort varies. The following theorem describes the most interesting case with a composed partial (total) negative application in the implying (implied) pattern, respectively.

Theorem 16 (implication of patterns). Let $C=\exists(\varnothing \hookrightarrow P, a c)$ and $C^{\prime}=\exists\left(\varnothing \hookrightarrow P^{\prime}, a c^{\prime}\right)$ be patterns with a composed total negative application condition $a c=\Lambda_{i \in I} \neg \exists\left(P \hookrightarrow^{x_{i}} X_{i}\right)$ and a composed partial negative application condition $a c^{\prime}=\Lambda_{j \in J} \neg \exists\left(P^{\prime} \sqcup_{j}^{x_{j}^{\prime}} X_{j}^{\prime}\right)$. Then $C^{\prime} \vDash C$, if the following conditions are fulfilled:

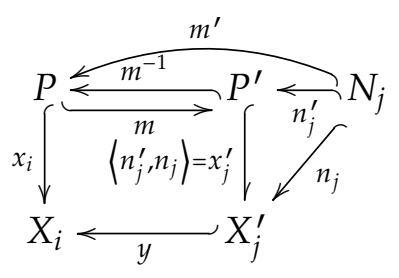

1. There exists a monomorphism $m: P \hookrightarrow P^{\prime}$ such that:

2. For each $i \in I$, there exists a $j \in J$ such that $n_{j}^{\prime}\left(\right.$ iface $\left.\left(x_{j}^{\prime}\right)\right) \subseteq m(P)$ and there exists a monomorphism $y: X_{j}^{\prime} \rightarrow X_{i}$ such that $y \circ n_{j}=x_{i} \circ m^{\prime}$, with $m^{\prime}=m^{-1} \circ n_{j}^{\prime}$.

Proof. Assuming that the above conditions hold, we have to show $\forall G\left(G \vDash C^{\prime} \Rightarrow\right.$ $G \vDash C)$.

Consider an arbitrary graph $G$ with $G \vDash C^{\prime}$. By definition of satisfaction, we have $i_{G} \vDash C^{\prime}$, implying the existence of a monomorphism $g^{\prime}: P^{\prime} \hookrightarrow G$ with $g^{\prime} \vDash \mathrm{ac}^{\prime}$. By assumption, there is a monomorphism $m: P \hookrightarrow P^{\prime}$. Then there exists a morphism $g: P \rightarrow G$ with $g=g^{\prime} \circ m$. Since the set of monomorphisms $\mathcal{M}$ is closed under composition, $g$ is a monomorphism. 


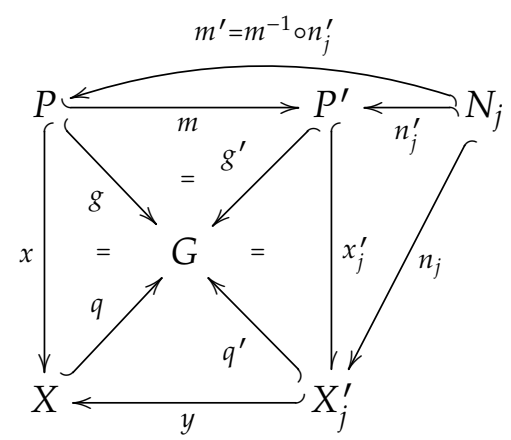

We will show $g \vDash$ ac by contradiction. Suppose $g \notin$ ac, implying the existence of a $x=x_{i}$ for some $i \in I$ and a corresponding monomorphism $q: X \hookrightarrow G$ with $g=q \circ x$, i.e. $g \not \neg \neg x$. By assumption, there is a monomorphism $y: X^{\prime} \hookrightarrow X$ with $X^{\prime}=X_{j}^{\prime}$ for some $j \in J$ and with $y \circ n_{j}=x \circ m^{\prime}$ and $m^{\prime}=m^{-1} \circ n_{j}^{\prime}$. Consequently, there is a monomorphism $q^{\prime}: X^{\prime} \hookrightarrow G$ with $q^{\prime}=q \circ y$. In addition, we have:

$$
\begin{array}{rrrr} 
& y \circ n_{j}= & x \circ m^{\prime} & \\
\Longrightarrow & q \circ y \circ n_{j}= & q \circ x \circ m^{\prime} & \\
\Longrightarrow & q^{\prime} \circ n_{j}= & g \circ m^{\prime} & \left(q^{\prime}=q \circ y \text { and } q \circ x=g\right) \\
\Longrightarrow & q^{\prime} \circ n_{j}= & g^{\prime} \circ m \circ m^{\prime} & \left(g=g^{\prime} \circ m\right) \\
\Longrightarrow & q^{\prime} \circ n_{j}= & g^{\prime} \circ m \circ m^{-1} \circ n_{j}^{\prime} & \left(m^{\prime}=m^{-1} \circ n_{j}^{\prime}\right) \\
\Longrightarrow & q^{\prime} \circ n_{j}= & g^{\prime} \circ n_{j}^{\prime} & \left(n_{j}^{\prime}\left(\text { iface }\left(x_{j}^{\prime}\right)\right) \subseteq m(P)\right)
\end{array}
$$

This implies $g^{\prime} \not \neg \exists x^{\prime}$ and therefore $g^{\prime} \not \neq \mathrm{ac}^{\prime}$. This is a contradiction, thus we have $g \vDash$ ac. With $g: P \hookrightarrow G$ and $g \vDash$ ac, we get $G \vDash C$, concluding the proof.

For patterns without negative application conditions, the theorem is also applicable as the second condition is trivially true. For cases where the implying pattern's partial negative application conditions do not satisfy the interface condition, a partial expansion of the implied pattern's condition is required, which requires additional computational effort.

In general, all cases can be transformed into a default case by expanding all composed partial negative application conditions into composed total negative application conditions with the Shift-construction. The comparison in that case is explained in Theorem 41 in Appendix B. The desired effect of the above theorem is to avoid this computationally expensive default case as often as possible.

This theorem only considers one implying pattern at a time. We also use an advanced implication check considering more complex relations between forbidden patterns and negative application conditions, such as implication of a single pattern 
by multiple patterns. The theory and implementation of such a check for the more general concept of nested conditions have already been introduced by Pennemann et. al. in [15]. Hence, we will not discuss our implementation here.

Besides graph constraints we will also encounter application conditions over a rule side, which can be interpreted as graph constraints as follows:

Lemma 17 (reduction to pattern). Let $a c=\exists\left(s: L \hookrightarrow S, a c_{S}\right)$ be an application condition over $L$ with acs being a composed partial negative application condition. For the reduction to a pattern $a c_{\varnothing}=\exists\left(i_{S}: \varnothing \hookrightarrow S, a c_{S}\right)$ of ac we have the following property: for each graph $G$ with a monomorphism $m: L \hookrightarrow G$ such that $m \vDash a c$, we have $G \vDash a c_{\varnothing}$.

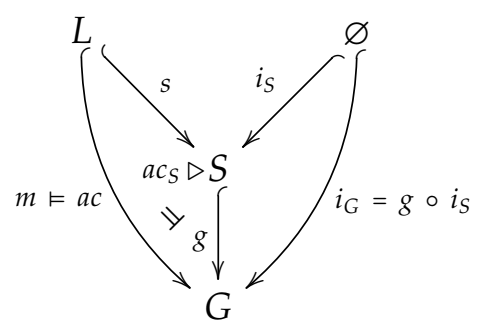

Proof. Consider an arbitrary graph $G$ with a monomorphism $m: L \hookrightarrow G$ such that $m \vDash$ ac. By definition of satisfiability, there exists a monomorphism $g: S \hookrightarrow G$ such that $g \vDash$ ac . For $i_{G}: \varnothing \hookrightarrow G$, we have $i_{G}=g \circ i_{S}$ and with $g \vDash$ ac , we have $i_{G} \vDash$ ac $\varnothing$. By definition of satisfiability, this implies $G \vDash \mathrm{ac}_{\varnothing}$, concluding the proof. 


\section{Inductive Invariant Checking}

Our inductive invariant checking algorithm consists of four basic steps:

1) From a composed forbidden pattern and a rule set, we create all pairs of individual forbidden patterns and rules to be analyzed on a per-pair basis. 2) We construct target patterns for each pair by applying the Shift- and PShift-constructions, such that each target pattern represents a satisfaction of a forbidden pattern after rule application. 3) From each target pattern, we construct a source pattern by applying the L-construction such that a source pattern is a representation for graphs before a rule application leads to a forbidden pattern. 4) We analyse source and target pattern pairs (counterexamples) for other forbidden or guaranteed patterns, which might invalidate the counterexample.

The first step of splitting a composed forbidden patterns into forbidden patterns for individual analysis is shown to be correct in the following lemma. It also explains the analysis of source and target patterns in step 4 .

Lemma 18. Given a composed forbidden pattern $\mathcal{F}=\bigwedge_{i \in I} \neg F_{i}$, a composed guaranteed pattern $\mathcal{G}=\wedge_{j \in J} \neg G_{j}$ and a typed graph transformation system $G T S=(T G, B), G T S$ is preserving $\mathcal{F}$ under $\mathcal{G}$ if, for each rule $b$ in $B$ it holds that:

$$
\forall G, H\left(\left(G \Rightarrow_{b} H\right) \Longrightarrow\left(\exists n\left(H \vDash F_{n}\right) \Rightarrow \exists k\left(H \vDash G_{k} \vee G \vDash G_{k} \vee G \vDash F_{k}\right)\right)\right)
$$

Proof. We can rearrange the formula from Definition 10 (with $j, k \in I$ and for each rule $b \in B$ ).

$$
\begin{aligned}
& \forall G, H((G \stackrel{b}{\Rightarrow} H) \Longrightarrow((G \vDash \mathcal{F} \wedge G \vDash \mathcal{G}) \Rightarrow(H \vDash \mathcal{F} \vee H \not \mathcal{G}))) \\
& \Longleftrightarrow \forall G, H((G \stackrel{b}{\Rightarrow} H) \Longrightarrow((H \not \mathcal{F} \wedge H \vDash \mathcal{G}) \Rightarrow(G \not \mathcal{F} \vee G \not \mathcal{G}))) \\
& \Longleftrightarrow \forall G, H((G \stackrel{b}{\Rightarrow} H) \Longrightarrow((H \not \mathcal{F}) \Rightarrow(H \not \mathcal{G} \vee G \not \mathcal{F} \vee G \not \mathcal{G}))) \\
& \Longleftrightarrow \forall G, H\left((G \stackrel{b}{\Rightarrow} H) \Longrightarrow\left(\left(H \vDash \bigvee_{i \in I} F_{i}\right) \Rightarrow\left(H \vDash \bigvee_{j \in J} G_{j} \vee G \vDash \bigvee_{j \in J} G_{j} \vee G \not \bigvee_{i \in I} F_{i}\right)\right)\right) \\
& \Longleftrightarrow \forall G, H\left((G \stackrel{b}{\Rightarrow} H) \Longrightarrow\left(\exists n\left(H \vDash F_{n}\right) \Rightarrow \exists k\left(H \vDash G_{k} \vee G \vDash G_{k} \vee G \vDash F_{k}\right)\right)\right)
\end{aligned}
$$

\subsection{Step 2: Construction of Target Patterns}

The second step in our inductive invariant checking algorithm is the creation of target patterns for each pair of a graph rule and a forbidden pattern such that the forbidden patterns occurs in the target pattern. Target patterns in general represent a set of graphs with a match for the right side of a specific graph rule. 
Definition 19 (target pattern). A target pattern over the right side $R$ of a rule $b$ is an application condition of the form tar $=$ false or tar $=\exists\left(t: R \leftrightarrow T, a c_{T}\right)$ with a composed partial negative application condition $a_{T}$ over $T$.

The set of graphs fulfilling such a target pattern is the set of graphs $H$ with a comatch $m^{\prime}: R \hookrightarrow H$ such that $m^{\prime} \vDash$ tar. For a rule $b$ in $B$ and a forbidden pattern $F$, we can create target patterns by transforming $F$ over the morphism $i_{R}: \varnothing \hookrightarrow R$ into an application condition over the right rule side $R$ :

Lemma 20 (creation of target patterns). Let $b=\left\langle(L \hookleftarrow K \hookrightarrow R), a c_{L}\right.$, true $\rangle$ be a rule and $F=\exists\left(i_{P}: \varnothing \hookrightarrow P, a c_{P}\right)$ a forbidden pattern with $a c_{P}$ and $a c_{L}$ being composed total negative application conditions. Let $b^{*}=\left\langle\left(L^{*} \hookleftarrow K^{*} \rightarrow R^{*}\right)\right\rangle$ be a reduced rule of $b$ with respective injective morphisms $r^{+}: R^{*} \hookrightarrow R, l^{+}: L^{*} \hookrightarrow L$, and $k^{+}: K^{*} \hookrightarrow K$. Then we have:

1. $\operatorname{Shift}\left(r^{+}, \operatorname{Shift}\left(i_{R^{*}}, \exists i_{P}\right)\right)=\bigvee_{j \in J} \exists t_{j}$.

2. $\vee_{j \in J}$ tar $_{j}$ is a set of target patterns for tar ${ }_{j}=\exists\left(t_{j}\right.$, PShift $\left.\left(t_{j}^{+}, \operatorname{Shift}\left(t_{k}^{\prime *}, a c_{p}\right)\right)\right)$.

3. For each graph $H$ and each monomorphism $h: R \hookrightarrow H$, it holds that $\exists j(j \in J \wedge h \vDash$ $\left.\operatorname{tar}_{j}\right) \Leftrightarrow H \vDash F$.

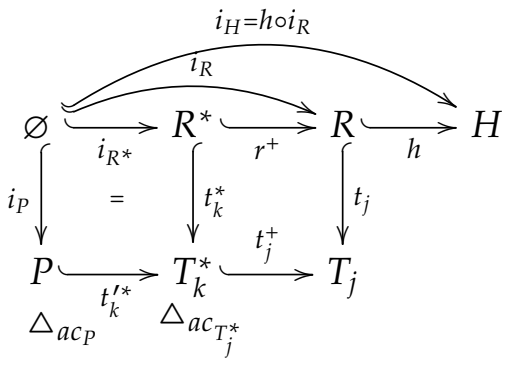

Proof. First, we will show that the construction yields the required structure.

According to the Shift-construction, we have $\operatorname{Shift}\left(i_{R^{*}}, \exists i_{P}\right)=\bigvee_{k \in K} \exists t_{k}^{*}$, where the index set $K$ depends on the number of injective and jointly surjective morphism pairs $\left(t_{k}^{*}, t_{k}^{\prime *}\right)$. Further, again because of the Shift-construction, we have $\operatorname{Shift}\left(r^{+}, \bigvee_{k \in K} \exists t_{k}^{*}\right)=\bigvee_{j \in J} \exists t_{j}$, where the index set $J$ now depends on the number of injective and jointly surjective morphism pairs $\left(t_{j}, t_{j}^{+}\right)$.

Since $\operatorname{ac}_{P}$ is a composed total negative application condition over $P$ and by Lemma $38, \mathrm{ac}_{T_{j}^{*}}=\operatorname{Shift}\left(t_{k}^{\prime *}, \mathrm{ac}_{p}\right)$ is a composed total negative application condition over a specific $T_{k}^{*}$. By the PShift-construction, $\operatorname{PShift}\left(t_{j}^{+}, \mathrm{ac}_{T_{j}^{*}}\right)$ is a composed partial negative application condition over $T_{j}$. Hence, $\operatorname{tar}_{j}=\exists\left(t_{j}, \operatorname{PShift}\left(t_{j}^{+}, \operatorname{Shift}\left(t_{k}^{\prime *}, \operatorname{ac}_{p}\right)\right)\right)$ is a target pattern over $R$ and thus $\bigvee_{j \in J} \operatorname{tar}_{j}$ is a set of target patterns over $R$. 
Second, by the Shift- and PShift-construction and by definition of satisfiability we have for each graph $H$ and each monomorphism $h: R \rightarrow H$ :

$$
\begin{array}{rlr} 
& H \vDash F & \\
\Longleftrightarrow i_{H} \vDash F & \text { (Definition 3) } \\
\Longleftrightarrow h \circ r^{+} \circ i_{R^{*}} \vDash F & \\
\Longleftrightarrow h \circ r^{+} \vDash \operatorname{Shift}\left(i_{R^{*}}, F\right) & \text { (Shift-construction) } \\
\Longleftrightarrow h \vDash \operatorname{Shift}\left(r^{+}, \operatorname{Shift}\left(i_{R^{*}}, F\right)\right) & \text { (Shift-construction) } \\
\Longleftrightarrow h \vDash \operatorname{Shift}\left(r^{+}, \bigvee_{k \in K} \exists\left(t_{k}^{*}, \operatorname{Shift}\left(t_{k}^{\prime *}, \operatorname{ac}_{P}\right)\right)\right) & \text { (Shift-construction) } \\
\Longleftrightarrow h \vDash \bigvee_{j \in J} \exists\left(t_{j}, \operatorname{Shift}\left(t_{j}^{+}, \operatorname{Shift}\left(t_{k}^{\prime *}, \operatorname{ac}_{P}\right)\right)\right) & \text { (Shift-construction) } \\
\Longleftrightarrow h \vDash \bigvee_{j \in J} \exists\left(t_{j}, \operatorname{PShift}\left(t_{j}^{+}, \operatorname{Shift}\left(t_{k}^{\prime *}, \operatorname{ac}_{P}\right)\right)\right) & \\
\Longleftrightarrow h \vDash \bigvee_{j \in J} \operatorname{tar}_{j} & \text { (Lemma 13) } \\
\Longleftrightarrow \exists j\left(j \in J \wedge h \vDash \operatorname{tar}_{j}\right) &
\end{array}
$$

In other words, we shift the exterior application condition $\left(\exists i_{P}\right.$ in step 1$)$ of the forbidden pattern to the right rule side, but its interior composed negative application condition ( $\mathrm{ac}_{P}$ in step 2 ) to a partial application condition using the reduced rule. Thus, we avoid creating a large number of morphism pairs when shifting the interior application condition to the complete right rule side.

In conclusion, for each morphism $h: R \rightarrow H$ the satisfaction of the forbidden pattern $F$ by a graph $H$ is equivalent to the existence of a target pattern $\operatorname{tar}_{j}$ satisfied by $h$. In other words, for each result of a possible rule application leading to a graph satisfying the forbidden pattern we have constructed a target pattern. Since target patterns (as shown above) are disjunctively combined, we can analyze each target pattern individually and compute its source pattern. By construction, we always have a finite number of target patterns.

\subsection{Step 3: Construction of Source Patterns}

For each target pattern constructed as described above, we try to generate a source pattern to represent the state before the application of the rule lead to the forbidden pattern. In general, we define source patterns analogously to target patterns as application conditions over the left side of a specific graph rule. 
Definition 21 (source pattern). A source pattern over the left side L of a rule $b$ is an application condition of the form $\operatorname{src}=$ false or $\operatorname{src}=\exists\left(s: L \hookrightarrow S, a c_{S}\right)$ with a composed partial negative application condition acs over $S$.

To construct source patterns to our target patterns, each target pattern is transformed into an application condition over the left rule side using the L-construction. Due to the nature of the L-construction, we create at most one source pattern per target pattern transformation.

Lemma 22 (creation of source patterns). Let tar $=\exists\left(t: R \rightarrow T, a_{T}\right)$ be a target pattern specific to a forbidden pattern $F$ and a rule $b=\left\langle(L \hookleftarrow K \hookrightarrow R), a c_{L}\right.$, true $\rangle$ with a reduced rule $b^{*}=\left\langle\left(L^{*} \hookleftarrow K^{*} \leftrightarrow R^{*}\right)\right\rangle$ of its plain rule and constructed as described above. Further, let $a c_{T}$ be a composed partial negative application condition $a c_{T}=P S h i f t\left(t^{+}, a c_{T}^{\prime}\right)$ with ac ${ }_{T}^{\prime}$ being a composed total negative application condition over $T^{*}$. Then we have:

1. $L(b, \exists t)$ is a source pattern and $L(b, \exists t)=$ false or $L(b, \exists t)=\exists s$.

2. For the latter case, src $=\exists\left(s, P S h i f t\left(s^{+}, L\left(b^{\prime}, a c_{T}^{\prime}\right)\right)\right)$ is a source pattern, with $b^{\prime}=$ $\left\langle S^{*} \hookleftarrow K^{\prime} \leftrightarrow T^{*}\right\rangle$ being the rule constructed via the pushout complement (1) and the pushout (2) and $s^{+}: S^{*} \rightarrow S$ such that $S \Rightarrow_{b^{\prime}, s^{+}, t^{+}} T$.

3. For each direct graph transformation $G \Rightarrow_{b, m, m^{\prime}} H: m \vDash s r c \Leftrightarrow m^{\prime} \vDash$ tar.

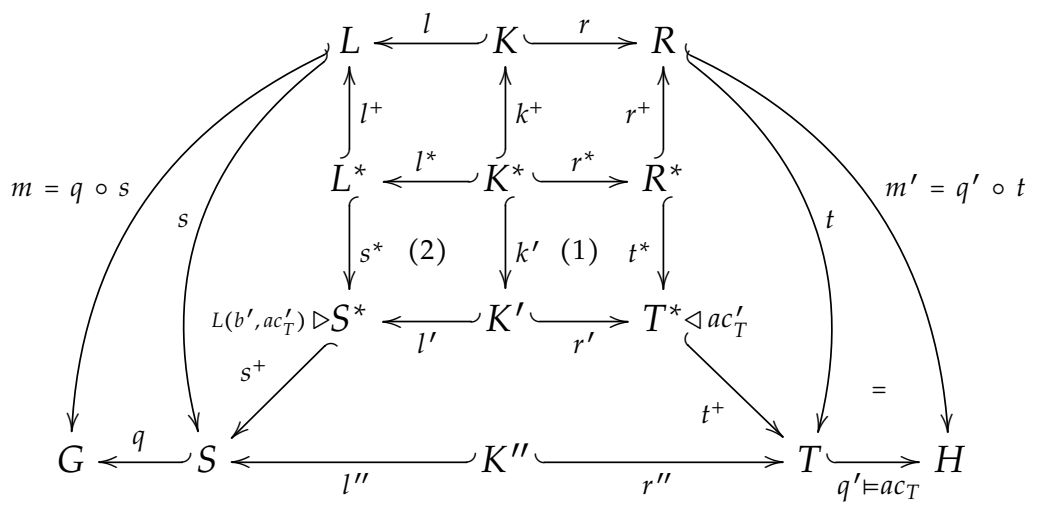

Proof. First, we will show that the construction yields the required structure.

We distinguish two cases:

1. $\langle r, t\rangle$ does not have a pushout complement. Then $\mathrm{L}(b, \exists t)=$ false. Since false is a source pattern, this case yields the required structure.

2. $\langle r, t\rangle$ has a pushout complement. Then, by constructing the pushout $\left(S, l^{\prime \prime}, s\right)$, we gain $\mathrm{L}(b, \exists t)=\exists(s: L \hookrightarrow S)$, which already is a source pattern. Further, $\mathrm{L}(b, \exists t)=\exists s$ implies $S \Rightarrow_{b, s, t} T$ and, because of the reduced rule $b^{*}$,

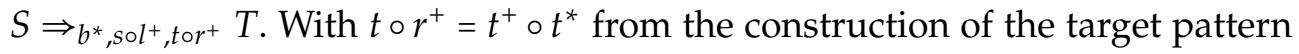


and, consequently, the existence of the pushout complement of $\left\langle t^{+} \circ t^{*}, r^{*}\right\rangle$, we can conclude the existence of the pushout complement of $\left\langle t^{*}, r^{*}\right\rangle$ to the pushout (1). This shows the existence of the rule $b^{\prime}=\left\langle S^{*} \hookleftarrow K^{\prime} \hookrightarrow T^{*}\right\rangle$.

Because of the construction of $b^{\prime}$ and since $S \Rightarrow_{b^{*}, \text { sol }} l^{+}$,or $r^{+} T$, we can conclude the existence of the pushout complement of $\left\langle r^{\prime}, t^{+}\right\rangle$and hence, the existence of an injective morphism $s^{+}: S^{*} \hookrightarrow S$ such that $S \Rightarrow_{b^{\prime}, s^{+}, t^{+}} T$. Then, $\operatorname{PShift}\left(s^{+}, \mathrm{L}\left(b^{\prime}, \mathrm{ac}_{T}^{\prime}\right)\right)$ is a composed partial negative application condition over $S$ and $\operatorname{src}=\exists\left(s, \operatorname{PShift}\left(s^{+}, \mathrm{L}\left(b^{\prime}, \mathrm{ac}_{T}^{\prime}\right)\right)\right)$ is a source pattern.

Furthermore, for each direct graph transformation $G \Rightarrow_{b, m, m^{\prime}} H$ we have:

$$
\begin{aligned}
& m^{\prime} \vDash \operatorname{tar} \\
& \Longleftrightarrow m^{\prime} \vDash \exists\left(t: R \hookrightarrow T, \mathrm{ac}_{T}\right) \\
& \Longleftrightarrow m^{\prime} \vDash \exists\left(t, \operatorname{PShift}\left(t^{+}, \mathrm{ac}_{T}^{\prime}\right)\right) \\
& \Longleftrightarrow m^{\prime} \vDash \exists\left(t, \operatorname{Shift}\left(t^{+}, \mathrm{ac}_{T}^{\prime}\right)\right) \\
& \left(\operatorname{ac}_{T}=\operatorname{PShift}\left(t^{+}, \operatorname{ac}_{T}^{\prime}\right)\right) \\
& \text { (Lemma 13) } \\
& \Longleftrightarrow \exists q^{\prime}\left(m^{\prime}=q^{\prime} \circ t \wedge q^{\prime} \vDash \operatorname{Shift}\left(t^{+}, \operatorname{ac}_{T}^{\prime}\right)\right) \\
& \Longleftrightarrow \exists q^{\prime}\left(m^{\prime}=q^{\prime} \circ t \wedge q^{\prime} \circ t^{+} \vDash \mathrm{ac}_{T}^{\prime}\right) \\
& \Longleftrightarrow \exists q\left(m=q \circ s \wedge q \circ s^{+} \vDash L\left(b^{\prime}, \mathrm{ac}_{T}^{\prime}\right)\right) \\
& \Longleftrightarrow \exists q\left(m=q \circ s \wedge q \vDash \operatorname{PShift}\left(s^{+}, L\left(b^{\prime}, \operatorname{ac}_{T}^{\prime}\right)\right)\right) \\
& \Longleftrightarrow m \vDash \exists\left(s: L \hookrightarrow S, \operatorname{PShift}\left(s^{+}, L\left(b^{\prime}, \operatorname{ac}_{T}^{\prime}\right)\right)\right) \\
& \Longleftrightarrow m \vDash \mathrm{src}
\end{aligned}
$$

Such a source pattern represents graphs before the application of the rule in question which leads to graphs satisfying the forbidden pattern. To also take left application conditions into account, they need to be transformed via Shift $\left(s, \mathrm{ac}_{L}\right)$ into conditions over the source pattern. For details, we refer to Lemma 42 in Appendix B.

In summary, the source and target patterns src and tar represent a correct rule application of a rule $b$ leading to the existence of the forbidden pattern $F$. To represent all possible rule applications, i.e. all graphs $G$ and $H$ with $G \Rightarrow_{b} H$, we need to consider all target patterns and their corresponding source patterns.

\subsection{Step 4: Analysis of Source Patterns and Counterexamples}

Each target pattern and corresponding source pattern specific to a rule and a forbidden pattern specify a counterexample for our inductive invariant, i.e. a situation where a rule application leads to the occurrence of a forbidden pattern $F_{i}$. 
To investigate whether this is indeed a violation of the inductive invariant $\mathcal{F}$ under $\mathcal{G}$, the following three conditions are considered:

1. The target pattern also violates the composed guaranteed pattern.

2. The source pattern violates the composed guaranteed pattern.

3. The source pattern violates the composed forbidden pattern.

Theorem 23 (inductive invariant checking). Let GTS be a graph transformation system and $\mathcal{F}=\bigwedge_{i \in I} \neg F_{i}$ and $\mathcal{G}=\bigwedge_{j \in J} \neg G_{j}$ be a composed forbidden and composed guaranteed pattern. Let, for each rule $b \in B$ and for $i \in I, \operatorname{src}_{b, i}\left(\operatorname{tar}_{b, i}\right)$ be the set of source (target) patterns constructed from the pair $\left(b, F_{i}\right)$ and $\operatorname{src}_{\varnothing, b, i}\left(\operatorname{tar}_{\varnothing, b, i}\right)$ be the set of these source (target) patterns reduced to graph constraints.

GTS preserves $\mathcal{F}$ under $\mathcal{G}$ if, for all reduced source patterns $\operatorname{src}_{\varnothing}$ created from a pair of a rule and a forbidden pattern $\left(b, F_{i}\right)$ and the corresponding reduced target pattern tar, one of the following conditions holds:

1. $\exists k\left(k \in J \wedge \operatorname{tar}_{\varnothing} \vDash G_{k}\right)$

2. $\exists k\left(k \in J \wedge \operatorname{src}_{\varnothing} \vDash G_{k}\right)$

3. $\exists k\left(k \in I \wedge \operatorname{src} \varnothing \vDash F_{k}\right)$

Proof. By Lemma 18 , the preservation of $\mathcal{F}$ under $\mathcal{G}$ by GTS is implied by (for each rule $b \in B$ and with $k, k^{\prime} \in I$ ):

$$
\forall G, H\left((G \stackrel{b}{\Rightarrow} H) \Longrightarrow\left(\exists j\left(H \vDash F_{j}\right) \Rightarrow \exists k\left(H \vDash G_{k} \vee G \vDash G_{k} \vee G \vDash F_{k}\right)\right)\right)
$$

Consider an arbitrary rule $b=\left\langle(L \hookleftarrow K \hookrightarrow R)\right.$, ac $_{L}$, true $\rangle$ from $B$ and graphs $G, H$ with $G \Rightarrow^{b} H$ and $\exists k^{\prime}\left(H \vDash F_{k^{\prime}}\right)$ with, for ease of reading, $F_{k^{\prime}}=F$. As a forbidden pattern, $F$ has the form $F=\exists\left(\varnothing \hookrightarrow P\right.$, ac $\left._{P}\right)$ with $\operatorname{ac}_{P}$ being a composed negative application condition.

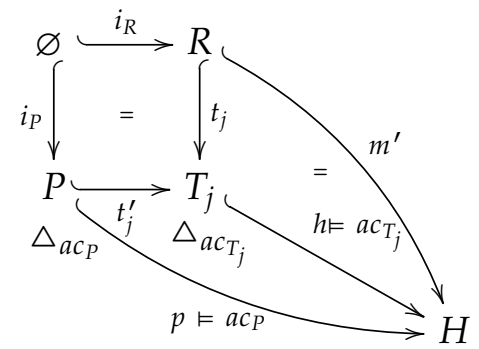

From $G \stackrel{b}{\Rightarrow} H$, we conclude the existence of a comatch $m^{\prime}: R \hookrightarrow H$. By construction of target patterns (Lemma 20), we have a number of target patterns $\operatorname{tar}_{j}$ and with $H \vDash F$ we gain $\exists j\left(j \in J \wedge m^{\prime} \vDash \operatorname{tar}_{j}\right)$. Consequently, there is a target pattern tar $=\exists(t$ : $\left.R \hookrightarrow T, \mathrm{ac}_{T}\right)$ with $m^{\prime} \vDash \operatorname{tar}$ for the specific $\operatorname{tar}_{j}=\operatorname{tar}$. 


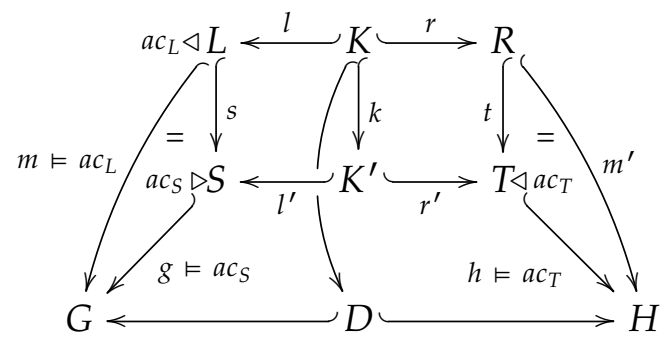

By construction of source patterns (Lemma 22), we get a source pattern src with $\operatorname{src} \in \operatorname{src}_{b, k^{\prime}}\left(\right.$ remember $\left.F=F_{k^{\prime}}\right)$. Note that src cannot be false, as this would contradict $G \Rightarrow_{b} H$. With $G \Rightarrow_{b, m, m^{\prime}} H$, where $m$ is a monomorphism $m: L \hookrightarrow G$, and $m^{\prime} \vDash$ tar, we get $m \vDash$ src.

By precondition, one of the following cases is true:

1. There is a $k \in J$ such that $\operatorname{tar}_{\varnothing} \vDash G_{k}$. Remember that $\operatorname{tar}_{\varnothing}=\exists\left(\varnothing \hookrightarrow T, \operatorname{ac}_{T}\right)$ is the reduction of tar to a pattern. By Lemma $17, m^{\prime} \vDash \operatorname{tar}$ implies $H \vDash \operatorname{tar}_{\varnothing}$ and with $\operatorname{tar}_{\varnothing} \vDash G_{k}$ and by Definition 15 we have $H \vDash G_{k}$, which concludes this case.

2. There is a $k \in J$ such that $\operatorname{src}_{\varnothing} \vDash G_{k}$. By Lemma $17, m \vDash \operatorname{src}$ implies $G \vDash \operatorname{src} \varnothing$ and with $\operatorname{src}_{\varnothing} \vDash G_{k}$ and by Definition 15 we have $G \vDash G_{k}$, which concludes this case.

3. There is a $k \in I$ such that $\operatorname{src}_{\varnothing} \vDash F_{k}$. By Lemma 17, $m \vDash \operatorname{src}$ implies $G \vDash \operatorname{src}_{\varnothing}$ and with $\operatorname{src}_{\varnothing} \vDash F_{k}$ and by Definition 15 we have $G \vDash F_{k}$, which concludes this case.

This shows that GTS preserves $\mathcal{F}$ under $\mathcal{G}$, if the condition from Theorem 23 holds. In other words, $\mathcal{F}$ is an inductive invariant for GTS under $\mathcal{G}$. The construction of target and source patterns and the verification of this condition by application of Theorem 16 is, in short, the essence of the Invariant Checking algorithm. On the other hand, source and target patterns not discarded by that conditions are counterexamples for the inductive invariant.

Example 24. Figure 2 shows a source and target pattern pair src and tar created from the forbidden pattern $F$ and rule in Examples 4 and 7 In tar, the condition $\exists t$ is one amalgamation of $F$ and the right rule side (Lemma 20. step 1 ); $\neg \exists p$ is the pattern's application condition transformed with PShift over $t^{+}$(Lemma 20, step 2). Since the forbidden pattern can be found in the source pattern $(\operatorname{src} \varnothing \vDash F)$, this counterexample is discarded by the analysis in Theorem 23 


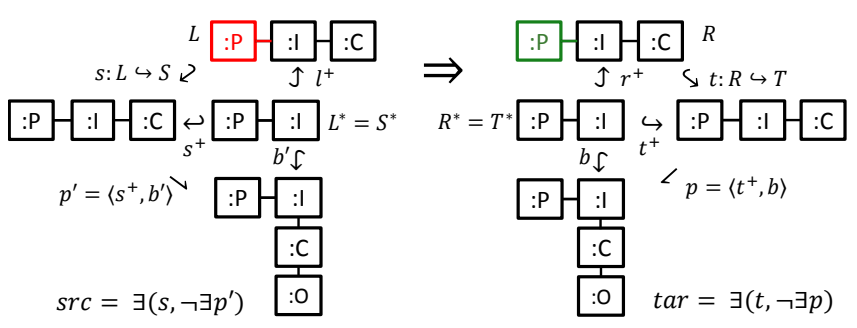

Figure 2: Source and target pattern pair created from a rule and a forbidden pattern

Because the implication checks (Theorems 16 and 23) compare only individual patterns and disregard more complex interdependencies and satisfiability of multiple patterns, this algorithm may still produce false negatives (i.e., spurious counterexamples). Our advanced implication check then serves to reduce this number and may also be applied to reduce the number of forbidden patterns to be analyzed by subsuming some of them. Since the general concept has already been introduced by Pennemann et. al. in [15], we do not discuss it here. However, our technique is safe in the sense that all violations will be reported. 


\section{Evaluation and Discussion}

To evaluate our results, we employ three case studies: The first example car platooning describes rules and constraints in a car platooning system. It was employed in the context of the SeekSat/ProCon tool [15] and was originally described in [12]. In order to conform to our restrictions it had to be adjusted, resulting in the addition of twelve new constraints. Our second and third case study are a simple and complex example for verification of behavior preservation of model transformations by bisimulation with the simple case initially employed by us in [10] and both examples described in [6]. In the first case (MT - Simple), behavioral equivalence between single lifelines and automata derived by a triple graph grammar (TGG) is proven. In the more complex example (MT - Complex), behavioral equivalence between sequence diagrams with multiple lifelines and networks of automata is proven. In both cases the check involves two inductive invariant checks: one for the TGG generating all possible model pairs and one for the Semantics of any possible pair of models to prove bisimilarity.

The first point of reference for our evaluation is our improved inductive invariant checker in its basic variant (invcheck-total). We also compare variants employing advanced implication checks (invcheck-total/impl), partial negative application conditions (invcheck-partial), and both (invcheck-partial/impl). On the other hand, the former version of our inductive invariant checker [2] only supported a restricted form of negative application conditions for constraints and rules and was thus not expressive enough for the considered case studies.

In addition, we will consider the SeekSat/ProCon tool [15, 11], which is able to prove correctness of graph programs with respect to pre- and postconditions specified as nested graph constraints. To verify an inductive invariant $(\mathcal{F})$ of a graph transformation system (GTS) with guaranteed constraints $(\mathcal{G})$, the equivalent check contains a graph program nondeterministically choosing a rule from GTS, the precondition $\{\mathcal{F} \wedge \mathcal{G}\}$ and the postcondition $\{\mathcal{F} \vee \neg \mathcal{G}\}$. While the technique behind SeekSat/ProCon is more expressive than our approach, we use this comparison to demonstrate the relevance of our more specialized tool for the verification of certain cases where that level of expressiveness is not needed.

Besides the evaluation of the case studies as a whole, we also want to study the impact of the complexity of the checking problem by considering the sum of all possible amalgamations between a forbidden pattern and the right side of a rule and the number of total negative application conditions for those amalgamations. To get more fine-grained results, we separated some examples into multiple cases by splitting postconditions $\left(\bigwedge_{i \in I} F_{i}\right) \vee \neg \mathcal{G}$ into less complex $i$ subproblems with postconditions $F_{i} \vee \neg \mathcal{G}$ or by considering rules in a set separately. 
Table 1: Complexity of verification problems and results of evaluated algorithms

\begin{tabular}{|c|c|c|c|c|c|c|c|c|c|c|c|c|}
\hline \multirow[b]{3}{*}{ Example } & \multirow{2}{*}{\multicolumn{2}{|c|}{ Characteristics }} & \multirow{2}{*}{\multicolumn{2}{|c|}{ SeekSat/ProCon }} & \multicolumn{4}{|c|}{ without advanced implication check } & \multicolumn{4}{|c|}{ with advanced implication check } \\
\hline & & & & & Invche & eck-total & Invchec & ck-partial & Invchecl & total/impl & Invcheck- & artial/imp \\
\hline & Check & Complexity & time (s) & result & time (s) & result & time (s) & result & time (s) & result & time $(\mathrm{s})$ & result \\
\hline MT - Simple - Semantics & subproblem & 4 & 20 & true & $<1$ & true & $<1$ & true & $<1$ & true & $<1$ & true \\
\hline MT - Simple - Semantics & subproblem & 4 & 20 & true & $<1$ & true & $<1$ & true & $<1$ & true & $<1$ & true \\
\hline MT - Complex - TGG & subproblem & 4 & $<1$ & true & $<1$ & true & $<1$ & true & $<1$ & true & $<1$ & true \\
\hline MT - Complex - TGG & subproblem & 4 & $<1$ & true & $<1$ & true & $<1$ & true & $<1$ & true & $<1$ & true \\
\hline MT - Complex - Semantics & subproblem & 5 & 10 & true & $<1$ & true & $<1$ & true & $<1$ & true & $<1$ & true \\
\hline MT - Complex - Semantics & subproblem & 5 & 9 & true & $<1$ & true & $<1$ & true & $<1$ & true & $<1$ & true \\
\hline MT - Simple - Semantics & subproblem & 11 & 40 & true & $<1$ & true & $<1$ & true & $<1$ & true & $<1$ & true \\
\hline MT - Complex - TGG & subproblem & 11 & $<1$ & true & $<1$ & true & $<1$ & true & $<1$ & true & $<1$ & true \\
\hline MT - Complex - Semantics & subproblem & 12 & & out of memory & & timeout & $<1$ & \begin{tabular}{|l|} 
false negatives \\
\end{tabular} & & timeout & $<1$ & true \\
\hline MT - Complex - Semantics & subproblem & 17 & 17 & true & $<1$ & false negatives & $<1$ & \begin{tabular}{|l|} 
false negatives \\
\end{tabular} & $<1$ & true & $<1$ & true \\
\hline MT - Complex - TGG & subproblem & 20 & & timeout & $<1$ & true & $<1$ & true & $<1$ & true & $<1$ & true \\
\hline MT - Simple - Semantics & subproblem & 30 & 20 & true & $<1$ & true & $<1$ & true & $<1$ & true & $<1$ & true \\
\hline MT - Simple - Semantics & subproblem & 70 & 40 & true & $<1$ & true & $<1$ & true & $<1$ & true & $<1$ & true \\
\hline MT - Complex - Semantics & subproblem & 72 & & timeout & $<1$ & false negatives & 1 & \begin{tabular}{|l|} 
false negatives \\
\end{tabular} & 1,5 & true & 1,5 & true \\
\hline MT - Simple - Semantics & subproblem & 78 & 6,5 & true & $<1$ & true & $<1$ & true & $<1$ & true & $<1$ & true \\
\hline MT - Complex - Semantics & subproblem & 188 & & out of memory & 1,5 & false negatives & 2,5 & \begin{tabular}{|l|} 
false negatives \\
\end{tabular} & $<1$ & true & $<1$ & true \\
\hline Car Platooning & subproblem & 258 & $<1$ & true & $<1$ & true & $<1$ & true & $<1$ & true & $<1$ & true \\
\hline Car Platooning & subproblem & 610 & $<1$ & true & $<1$ & true & $<1$ & true & $<1$ & true & $<1$ & true \\
\hline MT - Simple - Semantics & subproblem & 807 & & timeout & $<1$ & true & $<1$ & true & $<1$ & true & $<1$ & true \\
\hline Car Platooning & complete & 947 & $<1$ & false & $<1$ & \begin{tabular}{|l|l|l|l|l|l} 
fals negatives \\
\end{tabular} & $<1$ & \begin{tabular}{|l|} 
false negatives \\
\end{tabular} & 3 & false & 3 & false \\
\hline MT - Simple - TGG & subproblem & 2778 & 220 & true & 1,5 & $\begin{array}{ll}\text { false negatives } \\
\end{array}$ & 1 & \begin{tabular}{|l|} 
false negatives \\
\end{tabular} & 1,5 & true & 1 & true \\
\hline MT - Simple - TGG & subproblem & 2778 & 226 & true & 1,25 & false negatives & 1 & \begin{tabular}{|l|} 
false negatives \\
\end{tabular} & 1,25 & true & 1 & true \\
\hline MT - Simple - Semantics & complete & 3870 & & timeout & 1,5 & true & 1 & true & 1,5 & true & 1 & true \\
\hline MT - Simple - TGG & complete & 5556 & 562 & true & 2 & \begin{tabular}{|l|l|} 
false negatives \\
\end{tabular} & 2 & \begin{tabular}{|l|} 
false negatives \\
\end{tabular} & 2,25 & true & 1,75 & true \\
\hline MT - Complex - Semantics & subproblem & 607312 & & out of memory & & \begin{tabular}{|l|} 
timeout \\
\end{tabular} & 90 & \begin{tabular}{|l|} 
false negatives \\
\end{tabular} & & timeout & $<1$ & true \\
\hline MT - Complex - Semantics & complete & 607500 & & out of memory & & timeout & 95 & \begin{tabular}{|l|} 
false negatives \\
\end{tabular} & & timeout & $<1$ & true \\
\hline MT - Complex - TGG & complete & 1817622 & & timeout & & timeout & - 100min & true & & timeout & $\sim 50 \mathrm{~min}$ & true \\
\hline
\end{tabular}

The experiments were executed on a computer with an Intel Core-i7-264oM processor with two cores at 2,8 GHz, $8 \mathrm{~GB}$ of main memory and running Eclipse 4.2.2 and Java 8 with a limit of 2 GB on Java heap space. All values were rounded and values under a second were not distingiuished. Timeout refers to a forced timeout issued by the tool (SeekSat/ProCon) or manual abortion (our tool) - for the related cases in our tool after more than two days of calculation. Out of memory means that memory exceeded the Java heap space limit of 2 GB.

Table 1 shows an overview of the verification of our complete examples (marked as complete; in gray) and a more detailed list of subproblems ordered by complexity (marked as subproblem), respectively. All algorithms perform comparably well for the car platooning example, with SeekSat/ProCon performing significantly better for the unadjusted version than our algorithms. However, for the other complete cases our tool terminates while SeekSat/ProCon does not.

It is important to note that the inductive invariant checker without advanced implication checks yields false negatives for certain subproblems. Even more importantly, these false negatives do not occur when using the variant with advanced implication checks. This demonstrates that the improvement in accuracy due to advancement in implication checks is indeed relevant for the case studies.

Further, the results demonstrate that the complex model transformation case study cannot be verified by the inductive invariant checker variants without partial negative application conditions, as these attempts were aborted after more than 
two days of calculation without a result. In contrast to that, a verification time of 100 minutes (for the longest case) when employing partial negative application conditions shows a drastic improvement in scalability for the considered more complex cases. The additional use of advanced implication checks does then not only eliminates false negatives, but, for one case, also halves the verification time, showing another notable effect on performance.

While these case studies show both our improvements and the relevance of verification for specifications that conform to our restrictions, the data is not complete and heterogeneous enough to derive claims for the general case. While SeekSat/ProCon's more general approach is also successfully applicable for specifications that are significantly more expressive, our tool has been optimized for a particular class of problems present in the two more complex case studies and their verification only succeeded with our tool. 


\section{Related Work}

As already discussed in Section 5, the SeekSat/ProCon tool [15, 11] is more general than our approach and thus is in principle capable of addressing the case studies. However, the limited scalability of the SeekSat/ProCon tool demonstrates that there is still a need for a tool optimized for a particular class of problems that scales up to the presented two more complex case studies.

For all other automated approaches that approach graph transformation systems with infinite state space [17, 5, 14, 4, 2, 13, 1], it holds that, in contrast to the approaches considered in the evaluation, they cannot be used for the case studies which require unrestricted negative application conditions: The model checking approach [17] employing abstraction based on the summarization in shape analysis and the model checking approach [5] employing a neighborhood abstraction, but both do not support negative application conditions for the constraints or rules. The tool Uncover [14] supports well-structured graph transformation systems that can only be established for negative application conditions which forbid the existence of edges but not of nodes. The Augur tool [13, 1], which constructs a overapproximation in form of a so-called Petri graph, also considers only graph transformation systems without negative application conditions. Finally, the RAVEN tool [4] can check only invariants for graph transformation systems without negative application conditions whose reachable graphs are accepted by a finite graph automaton. Since two of our case studies describe reachable graphs by TGGs, they cannot be covered by a finite graph automaton.

For additional discussion of related work with respect to the general concept of inductive invariants, we refer to the respective section in [2]. 


\section{Conclusion and Future Work}

In this paper, we presented several improvements for the inductive invariant checker for graph transformation systems introduced in [2]. Support for more expressive negative application conditions in constraints and rules was shown to be necessary to address the considered case studies at all. The introduction of partial negative application conditions allowed avoiding the explicit representation of a large number of application conditions, which considerably improved scalability. The addition of advanced implication checks improved the accuracy, so that no false negatives are reported for the case studies.

In addition we demonstrated the outlined improvements by means of three case studies and compared our approach for a restricted class of problems with an existing tool that targets more general problems. For the more complex problems considered, our approach was still able to check them; the other tool was not.

While the results are promising, the evaluation also raises a number of possible future directions such as employing even more partial shifts in our constructions, and experimenting with the parallel execution of alternative strategies.

\section{Acknowledgments}

We would like to thank the group of Annegret Habel, in particular the authors of the SeekSat/ProCon tool [15], for allowing us to do the comparison and Leen Lambers for her work on behavior preservation of model transformations.

This work was partially developed in the course of the project Correct Model Transformations II (GI 765/1-2), which is funded by the Deutsche Forschungsgemeinschaft. 


\section{References}

[1] P. Baldan, A. Corradini, and B. König. A Static Analysis Technique for Graph Transformation Systems. In Proc. CONCUR, volume 2154 of LNCS, pages 381-395. Springer, 2001.

[2] B. Becker, D. Beyer, H. Giese, F. Klein, and D. Schilling. Symbolic Invariant Verification for Systems with Dynamic Structural Adaptation. In Proc. of the 28th International Conference on Software Engineering (ICSE), Shanghai, China. ACM Press, 2006.

[3] B. Becker, L. Lambers, J. Dyck, S. Birth, and H. Giese. Iterative Development of Consistency-Preserving Rule-Based Refactorings. In J. Cabot and E. Visser, editors, Theory and Practice of Model Transformations, Fourth International Conference, ICMT 2011, Zurich, Switzerland, June 27-28, 2011. Proceedings, volume 6707 of Lecture Notes in Computer Science, pages 123-137. Springer / Heidelberg, 2011.

[4] C. Blume, H. Bruggink, D. Engelke, and B. König. Efficient Symbolic Implementation of Graph Automata with Applications to Invariant Checking. In H. Ehrig, G. Engels, H.-J. Kreowski, and G. Rozenberg, editors, Graph Transformations, volume 7562 of Lecture Notes in Computer Science, pages 264-278. Springer Berlin Heidelberg, 2012.

[5] I. B. Boneva, J. Kreiker, M. E. Kurban, A. Rensink, and E. Zambon. Graph abstraction and abstract graph transformations (Amended version). Technical Report TR-CTIT-12-26, Centre for Telematics and Information Technology, University of Twente, Enschede, October 2012.

[6] J. Dyck, H. Giese, and L. Lambers. Automatic Verification of Behavior Preservation for Model Transformation via Invariant Checking. Technical report, Hasso Plattner Institute at the University of Potsdam, Germany, 2015. (forthcoming).

[7] H. Ehrig, K. Ehrig, U. Prange, and G. Taentzer. Fundamentals of Algebraic Graph Transformation (Monographs in Theoretical Computer Science. An EATCS Series). Springer-Verlag New York, Inc., Secaucus, NJ, USA, 2006.

[8] H. Ehrig, U. Golas, A. Habel, L. Lambers, and F. Orejas. M-Adhesive Transformation Systems with Nested Application Conditions, Part 1: Parallelism, Concurrency and Amalgamation. Mathematical Structures in Computer Science, volume 24, 2014 . 
[9] A. H. Ghamarian, M. J. de Mol, A. Rensink, E. Zambon, and M. V. Zimakova. Modelling and analysis using GROOVE. International journal on software tools for technology transfer, 14(1):15-40, February 2012.

[10] H. Giese and L. Lambers. Towards Automatic Verification of Behavior Preservation for Model Transformation via Invariant Checking. In H. Ehrig, G. Engels, H. Kreowski, and G. Rozenberg, editors, Proceedings of Intern. Conf. on Graph Transformation (ICGT 2012), volume 7562 of LNCS, pages 249-263. Springer, 2012.

[11] A. Habel and K.-H. Pennemann. Correctness of high-level transformation systems relative to nested conditions. Mathematical Structures in Computer Science, 19:1-52, 2009.

[12] A. Hsu, F. Eskafi, S. Sachs, and P. Varaiya. The design of platoon maneuver protocols for IVHS. Technical Report UCBITS-PRR-91-6, University of California, Berkley, 1991.

[13] B. König and V. Kozioura. Augur 2 - A New Version of a Tool for the Analysis of Graph Transformation Systems. Electronic Notes in Theoretical Computer Science, 211:201-210, 2008. Proceedings of the Fifth International Workshop on Graph Transformation and Visual Modeling Techniques (GT-VMT 2006).

[14] B. König and J. Stückrath. A General Framework for Well-Structured Graph Transformation Systems. In P. Baldan and D. Gorla, editors, CONCUR 2014 - Concurrency Theory, volume 8704 of Lecture Notes in Computer Science, pages 467-481. Springer Berlin Heidelberg, 2014.

[15] K.-H. Pennemann. Development of Correct Graph Transformation Systems. PhD thesis, Department of Computing Science, University of Oldenburg, 2009.

[16] A. Schmidt and D. Varró. CheckVML: A Tool for Model Checking Visual Modeling Languages. In Proceedings of the 6th International Conference on the Unified Modeling Language, UML2003, volume 2863 of Lecture Notes in Computer Science, pages 92-95. Springer Verlag, 2003.

[17] D. Steenken. Verification of Infinite-State Graph Transformation Systems via Abstraction. PhD thesis, University of Paderborn, March 2015. 


\section{A. Additional Definitions}

This section lists additional basic definitions and facts from [7] and [8] that are not included in the main report. For omitted proofs, we refer to the respective sources.

Many of the following notions also exist in more general forms which can be described by the concepts of category theory. However, this report is only concerned

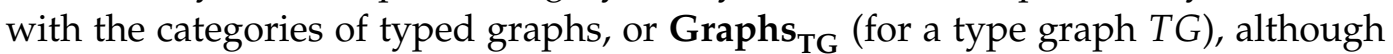
generalization of its concepts may be possible. Hence, some of the more general definitions, facts and lemmas taken from [7] and [8] have been slightly adjusted to fit this scope.

Definition 25 (graph [7]). A graph $G=(V, E, s, t)$ consists of a set $V$ of nodes (also called vertices), a set $E$ of edges and two functions $s, t: E \rightarrow V$, the source and target functions.

Definition 26 (graph morphism [7]). Given graphs $G_{1}, G_{2}$ with $G_{i}=\left(V_{i}, E_{i}, s_{i}, t_{i}\right)$ for $i=1,2$, a graph morphism $f: G_{1} \rightarrow G_{2}, f=\left(f_{V}, f_{E}\right)$ consists of two functions $f_{V}: V_{1} \rightarrow$ $V_{2}$ and $f_{E}: E_{1} \rightarrow E_{2}$ that preserve the source and target functions, i.e. $f_{V} \circ s_{1}=s_{2} \circ f_{E}$ and $f_{V} \circ t_{1}=t_{2} \circ f_{E}$.

A graph morphism $f$ is injective (or surjective) if both functions $f_{V}, f_{E}$ are injective (or surjective, respectively); $f$ is called isomorphic (or an isomorphism) if it is bijective, which means both injective and surjective. We use the terms injective morphism and monomorphism synonymously and describe the set of all monomorphisms with $\mathcal{M}$.

Fact 27 (composition of graph morphisms [7]). Given two graph morphisms $f=$ $\left(f_{V}, f_{E}\right): G_{1} \rightarrow G_{2}$ and $g=\left(g_{V}, g_{E}\right): G_{2} \rightarrow G_{3}$, the composition $g \circ f=\left(g_{V} \circ f_{V}, g_{E} \circ f_{E}\right)$ : $G_{1} \rightarrow G_{3}$ is again a graph morphism.

Definition 28 (typed graph and typed graph morphism [7]). A type graph is a distinguished graph $T G=\left(V_{T G}, E_{T G}, s_{T G}, t_{T G}\right)$. $V_{T G}$ and $E_{T G}$ are called the vertex and the edge type alphabets, respectively.

A tuple $(G$, type) of a graph $G$ together with a graph morphism type: $G \rightarrow T G$ is then called $a$ typed graph.

Given typed graphs $G_{1}^{T}=\left(G_{1}\right.$, type $\left._{1}\right)$ and $G_{2}^{T}=\left(G_{2}\right.$, type $\left.e_{2}\right)$, a typed graph morphism $f: G_{1}^{T} \rightarrow G_{2}^{T}$ is a graph morphism $f: G_{1} \rightarrow G_{2}$ such that type ${ }_{2} \circ f=$ type $_{1}$.

Definition 29 (jointly surjective (adjusted from [7])). A graph morphism pair $\left(e_{1}, e_{2}\right)$ with $e_{i}: A_{i} \rightarrow B(i=1,2)$ is called jointly surjective if, for all $g, h: B \rightarrow C$ with $g \circ e_{i}=h \circ e_{i}$ for $i=1,2$, we have $g=h$.

Definition 30 (pushout [7]). Given morphisms $f: A \rightarrow B$ and $g: A \rightarrow C$ in a category C, a pushout $\left(D, f^{\prime}, g^{\prime}\right)$ over $f$ and $g$ is defined by 
- a pushout object $D$ and

- morphisms $f^{\prime}: C \rightarrow D$ and $g^{\prime}: B \rightarrow D$ with $f^{\prime} \circ g=g^{\prime} \circ f$

such that the following unsiversal property is fulfilled: For all objects $X$ and morphisms $h: B \rightarrow X$ and $k: C \rightarrow X$ with $k \circ g=h \circ f$, there is a unique morphism $x: D \rightarrow X$ such that $x \circ g^{\prime}=h$ and $x \circ f^{\prime}=k$.

Fact $3 \mathbf{1}$ (pushouts [7]). Given morphisms $f: A \rightarrow B$ and $g: A \rightarrow C$ and a pushout $\left(D, f^{\prime}, g^{\prime}\right)$ over $f$ and $g$, we have the following properties:

1. If $f$ is injective (or surjective), then $f^{\prime}$ is also injective (or surjective, respectively).

2. The pair $\left(f^{\prime}, g^{\prime}\right)$ is jointly surjective, i.e. for each $x \in D$ there is a preimage $b \in B$ with $g^{\prime}(b)=x$ or $c \in C$ with $f^{\prime}(c)=x$.

3. If $f$ is injective and $x \in D$ has preimages $b \in B$ and $c \in C$ with $g^{\prime}(b)=f^{\prime}(c)=x$, then there is a unique preimage $a \in A$ with $f(a)=b$ and $g(a)=c$.

4. If $f$ and hence also $f^{\prime}$ is injective, then $D$ is isomorphic to $D^{\prime}=C \cup B \backslash f(A)$.

When trying to determine the applicability of graph rules, as will be explained in the following, the reverse construction of a pushout is required [7]. This notion is defined as the pushout complement.

Definition 32 (pushout complement [7]). Given morphisms $f: A \rightarrow B$ and $n: B \rightarrow D$, then $A \stackrel{m}{\rightarrow} C \stackrel{g}{\rightarrow} D$ is the pushout complement of $f$ and $n$ if $(1)$ below is a pushout:

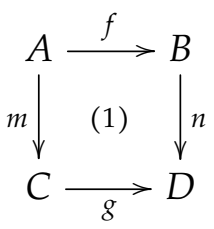

Fact 33 (application of graph rules [8]). The application of a graph rule $b=\left\langle p, a c_{L}, a c_{R}\right\rangle$ amounts to the following steps:

1. Find a match $m: L \rightarrow G$ satisfying $a_{L}$ as well as the gluing condition:

Dangling condition: No edge in $G \backslash m(L)$ is incident to a node in $m(L) \backslash m(K)$.

Identification condition: For all distinct items $x, y \in L, m(x)=m(y)$ only if $x, y \in K$. (This condition is understood to hold separately for nodes and edges.)

2. Remove $m(L \backslash K)$ from $G$, yielding a graph $D$ and add $R \backslash K$, yielding a graph $H$.

3. Check whether the comatch $m^{\prime}: R \rightarrow H$ satisfies $a c_{R}$.

Due to our restriction on injective matches and comatches, we do not need to consider the identification condition. Note that the satisfiability of the gluing condition is equivalent to the existence of the context graph $D$ with the pushout (1), i.e. the existence of the corresponding pushout complement [7]. 
Definition 34 (graph transformation system [7]). A graph transformation system GTS $=(B)$ consists of a set of graph rules $B$. A typed graph transformation system $G T S=(B, T G)$ consists of a set of gprah rules $B$ and a type graph $T G$. 


\section{B. Additional Lemmas and Constructions}

This section lists additional lemmas and constructions, which are not included in the main report and are mainly relevant for proofs.

Construction 35 (construction of reduced rules). Given a rule $b=\langle L \leftrightarrow K \hookrightarrow R\rangle, a$ reduced rule $b^{*}=\left\langle L^{*} \hookleftarrow K^{*} \hookrightarrow R^{*}\right\rangle$ of $b$ can be constructed as follows:

1. Create $K^{*}$ as a subset of $K$ containing at least all nodes $n$ that fulfil at least one of the following two conditions:

a) The image of $n$ under $r$ is adjacent to an edge in $R \backslash r(K)$.

b) The image of $n$ under $l$ is adjacent to an edge in $L \backslash l(K)$.

2. Create $K^{*} \stackrel{r^{*}}{\rightarrow} R^{*} \stackrel{r^{+}}{\rightarrow} R$ as the pushout complement of $k^{+}$and $r$.

3. Create $K^{*} \stackrel{l^{*}}{\hookrightarrow} L^{*} \stackrel{r^{+}}{\hookrightarrow} L$ as the pushout complement of $k^{+}$and $l$.

Proof. We have to show that for all graphs $G, H$ and all monomorphisms $m, m^{\prime}$, it holds that $G \Rightarrow_{b, m, m^{\prime}} H \Leftrightarrow G \Rightarrow_{b^{*}, m^{\circ} l^{+}, m^{\prime} \circ r^{+}} H$. Note that both pushout complements (1) and (2) exist by construction of $K^{*}$.

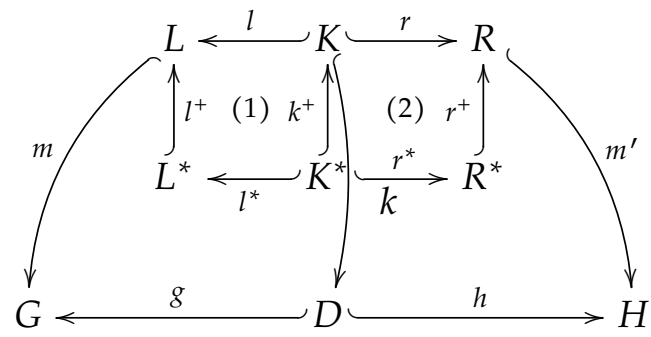

Only if. Since (1) is a pushout by construction and $(G, g, m)$ is a pushout by precondition, by pushout composition, $(1)+(G, g, m)$, or $\left(G, g, m \circ l^{+}\right)$is a pushout. Since (2) is a pushout by construction and $\left(H, h, m^{\prime}\right)$ is a pushout by precondition, by pushout composition, $(2)+\left(H, h, m^{\prime}\right)$, or $\left(H, h, m^{\prime} \circ r^{+}\right)$is a pushout, so that $G \Rightarrow_{b^{*}, m o l^{+}, m^{\prime} \circ r^{+}} H$.

If. Since $\left(G, g, m \circ l^{+}\right)$, or $(1)+(G, g, m)$ is a pushout by precondition and (1) is a pushout by construction, by pushout decomposition $(G, g, m)$ is a pushout. Since $\left(H, h, m^{\prime} \circ r^{+}\right)$, or $(2)+\left(H, h, m^{\prime}\right)$ is a pushout by precondition and (2) is a pushout by construction, by pushout decomposition $\left(H, h, m^{\prime}\right)$ is a pushout, so that $G \Rightarrow_{b, m, m^{\prime}} H$. This conlcudes the proof.

The following lemmas and constructions are concerned with the transformation on application conditions over morphisms and rules. 
Lemma 36 (shift of application conditions over morphisms, adjusted from [8]). There is a Shift-construction such that, for each application condition ac over $P$ and for each morphism $b: P \rightarrow P^{\prime}$, Shift transforms ac via $b$ into an application condition with only monomorphisms Shift $(b, a c)$ over $P^{\prime}$ such that, for each morphism $n: P^{\prime} \rightarrow H$ with $n \in \mathcal{M}, n \circ b \vDash a c \Leftrightarrow n \vDash \operatorname{Shift}(b, a c)$.

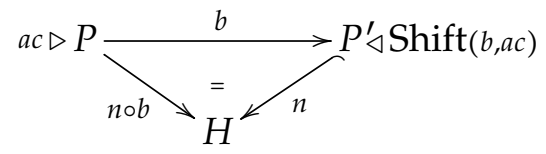

Construction (Shift-construction, adjusted from [8]). The Shift-construction is inductively defined as follows:

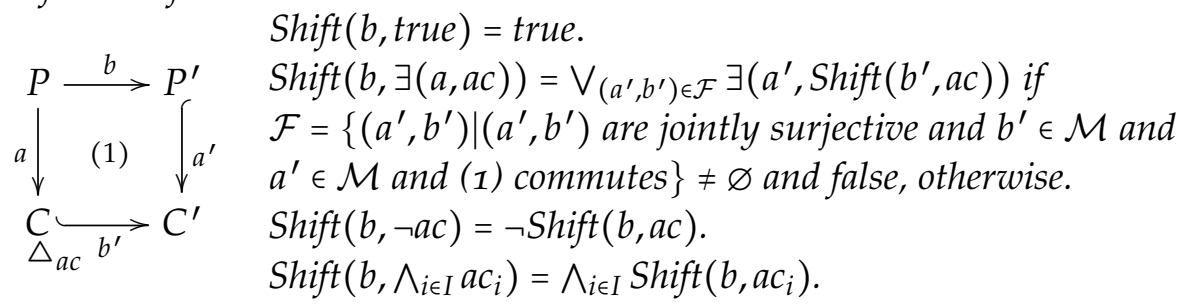

Proof. The proof in [8] can be adjusted to the special case where only monomorphisms are concerned.

Lemma 37 (shift of application conditions over rules [8, [1]]). There is a L-construction such that, for each rule $b$ and each application condition ac over $R, L$ transform $a c$ via $b$ into $L(b, a c)$ oder $L$ such that, for each direct transformation $G \Rightarrow_{b, m, m^{\prime}} H$, we have $m \vDash L(b, a c) \Leftrightarrow m^{\prime} \vDash a c$.

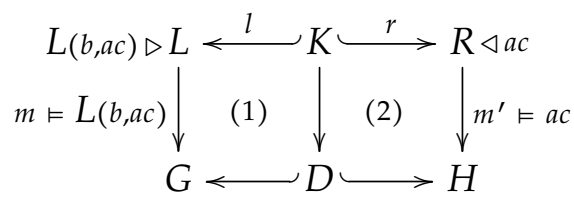

Construction (L-construction [8, 11]). The L-construction is inductively defined: $L(b$, true $)=$ true.

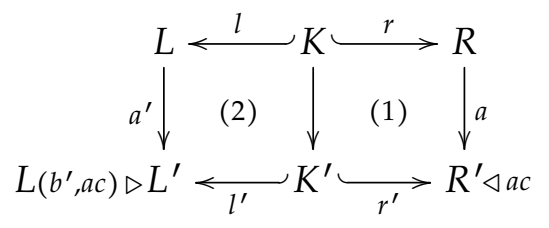
$L(b, \exists(a, a c))=\exists\left(a^{\prime}, L\left(b^{\prime}, a c\right)\right)$ with $b^{\prime}=$ $\left\langle L^{\prime} \hookleftarrow K^{\prime} \hookrightarrow R^{\prime}\right\rangle$ constructed via the pushouts (1) and (2)) if $\langle r, a\rangle$ has a pushout complement (1) and false, otherwise. $L(b, \neg a c)=\neg L(b, a c)$. $L\left(b, \wedge_{i \in I} a c_{i}\right)=\bigwedge_{i \in I} L\left(b, a c_{i}\right)$.

The following two lemmas are concerned with the structure-preserving properties of both the Shift- and L-construction with respect to composed total negative application conditions. 
Lemma 38. Let $a c=\exists\left(a: P \hookrightarrow C, a c_{C}\right)$ with graphs $P, C$, an application condition $a c_{C}$ and a monomorphism $a: P \hookrightarrow C$ be an application condition. For each graph $P^{\prime}$ and each monomorphism $b: P \hookrightarrow P^{\prime}$, there is a jointly surjective morphism pair $\left(b^{\prime}, a^{\prime}\right)$ with $a^{\prime} \in \mathcal{M}$ and $b^{\prime} \in \mathcal{M}$ such that $b^{\prime} \circ a=a^{\prime} \circ b$. Also, Shift $(b, a c)$ does not produce the trivial case false.

Given a composed total negative application condition $a c_{N}=\wedge_{i \in I} \neg \exists\left(x_{i}: N \hookrightarrow X_{i}\right)$ with graphs $N, X_{i}$, monomorphisms $x_{i}$ and an index set $I$, for each graph $N^{\prime}$ and each monomorphism $n: N \hookrightarrow N^{\prime}$ the application condition $a c_{N}^{\prime}=\operatorname{Shift}\left(n, a c_{N}\right)$ is a composed total negative application condition.

Proof. Given ac $=\exists\left(a: P \hookrightarrow C\right.$, ac $\left._{C}\right)$ and a monomorphism $b: P \hookrightarrow P^{\prime}$, we can construct the pushout $\left(C^{\prime}, b^{\prime}, a^{\prime}\right)$ over $b, a$. By Fact 31 . we have the pushout object $C^{\prime}$ and the jointly surjective morphism pair $\left(b^{\prime}, a^{\prime}\right)$ with $a^{\prime} \in \mathcal{M}$ and $b^{\prime} \in \mathcal{M}$ and $b^{\prime} \circ a=a^{\prime} \circ b$. Considering Lemma 36, we have $\mathcal{F}=\left\{\left(a^{\prime}, b^{\prime}\right) \in \mathcal{E}^{\prime} \mid b^{\prime} \in \mathcal{M}\right.$ and $a^{\prime} \in$ $\mathcal{M}$ and (1) commutes $\} \neq \varnothing$. Therefore, $\operatorname{Shift}(b$, ac $)$ does not produce the trivial case false (see the construction to Lemma 36). This shows the first property and can be extended to Boolean combinations of application conditions.

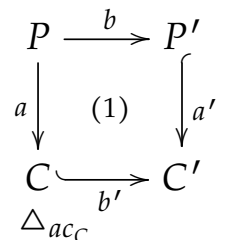

For a composed total negative application condition $\operatorname{ac}_{N}=\bigwedge_{i \in I} \neg \exists\left(x_{i}: N \hookrightarrow X_{i}\right)$, consider the following diagram and equations, with $\mathcal{F}_{i}=\left\{\left(x_{i}^{\prime}, n_{i}^{\prime}\right) \in \mathcal{E}^{\prime} \mid x_{i}^{\prime} \in \mathcal{M}\right.$ and $n_{i}^{\prime} \in$ $\mathcal{M}$ and (1) commutes\} (see construction to Lemma 36 ).

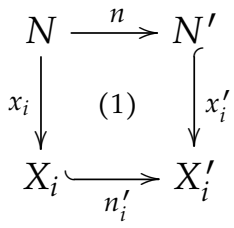




$$
\begin{array}{rlr}
\operatorname{Shift}\left(n, \operatorname{ac}_{N}\right) & =\operatorname{Shift}\left(n, \bigwedge_{i \in I} \neg \exists\left(x_{i}: N \hookrightarrow X_{i}\right)\right) \\
& =\bigwedge_{i \in I}\left(\operatorname{Shift}\left(n, \neg \exists\left(x_{i}: N \hookrightarrow X_{i}\right)\right)\right) & \text { (Lemma 36) } \\
& =\bigwedge_{i \in I}\left(\neg \operatorname{Shift}\left(n, \exists\left(x_{i}: N \hookrightarrow X_{i}\right)\right)\right) & \text { (Lemma 36) } \\
& =\bigwedge_{i \in I}\left(\neg \bigvee_{\left(x_{i}^{\prime}, n_{i}^{\prime}\right) \in \mathcal{F}_{i}} \exists\left(x_{i}^{\prime}, \operatorname{Shift}\left(n_{i}^{\prime}, \text { true }\right)\right)\right) & \left(\mathcal{F}_{i} \neq \varnothing,\right. \text { see proof above) } \\
& =\bigwedge_{i \in I}\left(\neg \bigvee_{\left(x_{i}^{\prime}, n_{i}^{\prime}\right) \in \mathcal{F}_{i}} \exists x_{i}^{\prime}\right) & \\
& =\bigwedge_{i \in I}\left(\bigwedge_{\left(x_{i}^{\prime}, n_{i}^{\prime}\right) \in \mathcal{F}_{i}} \neg \exists x_{i}^{\prime}\right) & \text { (De Morgan's laws) }
\end{array}
$$

As a conjunction of conjunctions of negative application conditions, $\operatorname{ac}_{N}^{\prime}=\operatorname{Shift}\left(n, \mathrm{ac}_{N}\right)$ is again a composed total negative application condition. This concludes the proof.

Lemma 39. Given a composed total negative application condition $a c=\bigwedge_{i \in I} \neg \exists\left(x_{i}: R \hookrightarrow\right.$ $\left.X_{i}\right)$, for each rule $b=\left\langle(L \hookleftarrow K \hookrightarrow R), a c_{L}\right.$, true $\rangle, L(b, a c)$ is a composed total negative application condition.

Proof. Consider the following diagram, illustrating the monomorphisms $x_{j}$ for which $\left\langle r, x_{j}\right\rangle$ has a pushout complement (1). We need to distinguish the cases where the pushout complement to (1) exists from the cases where it does not. Hence, we separate the index set $I$ in two disjoint sets $J \subseteq I$ and $K \subseteq I$ with $J \cap K=\varnothing$ and $J \cup K=I$ such that $J=\left\{i \in I \mid\left\langle r, x_{i}\right\rangle\right.$ has a pushout complement (1) $\}$ and $K=\left\{i \in I \mid\left\langle r, x_{i}\right\rangle\right.$ does not have a pushout complement (1) $\}$.

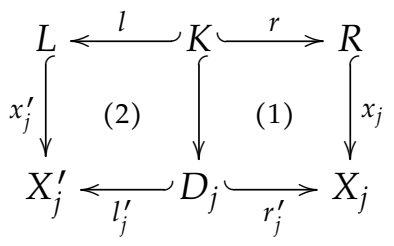




$$
\begin{aligned}
\mathrm{L}(b, \mathrm{ac}) & =\mathrm{L}\left(b, \bigwedge_{i \in I} \neg \exists\left(x_{i}: N \hookrightarrow X_{i}\right)\right) \\
& =\bigwedge_{i \in I}\left(\mathrm{~L}\left(b, \neg \exists\left(x_{i}: N \hookrightarrow X_{i}\right)\right)\right. \\
& =\bigwedge_{i \in I}\left(\neg \mathrm{L}\left(b, \exists\left(x_{i}: N \hookrightarrow X_{i}\right)\right)\right. \\
& =\bigwedge_{j \in J}\left(\neg \mathrm { L } ( b , \exists ( x _ { j } : N \hookrightarrow X _ { j } ) ) \wedge \bigwedge _ { k \in K } \left(\neg \mathrm{L}\left(b, \exists\left(x_{k}: N \hookrightarrow X_{k}\right)\right)\right.\right. \\
& =\bigwedge_{j \in J}\left(\neg \exists\left(x_{j}^{\prime}: N \hookrightarrow X_{j}^{\prime}\right)\right) \wedge \bigwedge_{k \in K}(\neg \text { false }) \\
& =\bigwedge_{j \in J}\left(\neg \exists\left(x_{j}^{\prime}: N \hookrightarrow X_{j}^{\prime}\right)\right) \wedge \text { true } \\
& =\bigwedge_{j \in J}\left(\neg \exists\left(x_{j}^{\prime}: N \hookrightarrow X_{j}^{\prime}\right)\right)
\end{aligned}
$$

If $J=\varnothing, \mathrm{L}(b$, ac $)=\bigwedge_{j \in J}\left(\neg \exists\left(x_{j}^{\prime}: N \rightarrow X_{j}^{\prime}\right)\right)$ is true, which is a combined negative application condition (Definition 8). If $J \neq \varnothing, \mathrm{L}(b, \mathrm{ac})=\bigwedge_{j \in J}\left(\neg \exists\left(x_{j}^{\prime}: N \hookrightarrow X_{j}^{\prime}\right)\right)$ has the structure required in Definition 8 , making it a composed total negative application condition.

The following lemma and construction offer a means of creating equivalent (with respect to a morphism) partial application conditions from total application conditions.

Lemma 40 (creation of partial application conditions). There is a PShift-construction such that, for each application condition ac over $P^{\prime}$ and for each morphism $p^{\prime}: P^{\prime} \hookrightarrow P$, PShift transforms ac via $p^{\prime}$ into a partial application condition PShift $\left(p^{\prime}\right.$, ac) over $P$ such that, for each morphism $n: P \hookrightarrow H$ with $n \in \mathcal{M}, n \circ p^{\prime} \vDash a c \Leftrightarrow n \vDash P S h i f t\left(p^{\prime}, a c\right)$.

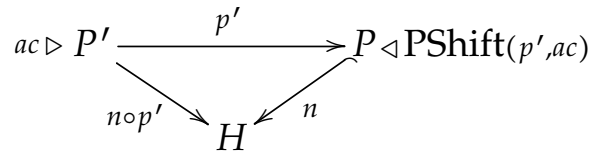

Construction (PShift-construction). The PShift-construction is defined as follows:

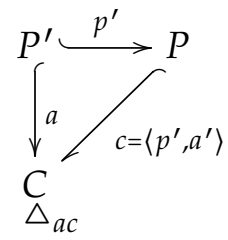

PShift $\left(p^{\prime}\right.$, true $)=$ true .

$\operatorname{PShift}\left(p^{\prime}, \exists(a, a c)\right)=\exists(c, a c)$ with $c=\left\langle p^{\prime}, a\right\rangle$.

$\operatorname{PShift}\left(p^{\prime}, \neg a c\right)=\neg \operatorname{PShift}\left(p^{\prime}, a c\right)$ and $\operatorname{PShift}\left(p^{\prime}, \wedge_{i \in I} a c_{i}\right)=$ $\bigwedge_{i \in I} \operatorname{PShift}\left(p^{\prime}, a c_{i}\right)$. 
Proof. (Adopted from Lemma 36 [8].) The statement is proven by structural induction:

Basis. For ac $=$ true, the statement holds.

Inductive step. For an application condition ac $=\exists\left(a, \mathrm{ac}^{\prime}\right)$, we have to show $n \circ p^{\prime} \vDash$ ac $\Leftrightarrow n \vDash \operatorname{PShift}\left(p^{\prime}, \exists\left(a, \mathrm{ac}^{\prime}\right)\right)$.

Only if. Let $n \circ p^{\prime} \vDash \exists\left(a, a^{\prime}\right)$. Consequently, there exists a monomorphism $q: C \hookrightarrow$ $H$ such that $n \circ p^{\prime}=q \circ a$ and $q \vDash$ ac. Thus, by satisfiability of partial application conditions, we have $n \vDash \exists(c$, ac $)$ with $c=\left\langle p^{\prime}, a\right\rangle$ and by construction we get $n \vDash$ $\operatorname{PShift}\left(p^{\prime}, \exists\left(a, \mathrm{ac}^{\prime}\right)\right)$.

If. Let $n \vDash \operatorname{PShift}\left(p^{\prime}, \exists\left(a, \mathrm{ac}^{\prime}\right)\right)$. By construction, we have $n \vDash \exists(c$, ac $)$ with $c=$ $\left\langle p^{\prime}, a\right\rangle$. By satisfiability of partial application conditions, we conclude the existence of a monomorphisms $q: C \hookrightarrow H$ such that $n \circ p^{\prime}=q \circ a$ and $q \vDash$ ac. Thus, we have $p^{\prime} \vDash \mathrm{ac}$, which concludes the inductive proof.

To avoid unnecessary computational effort, partial application conditions can be used instead of shifting total application conditions. The following lemma describes this equivalence.

As opposed to the theorem presented in the short version, the following theorem establishes a sufficient condition for checking implication of patterns for patterns with composed total negative application conditions.

Theorem $4 \mathbf{1}$ (implication of patterns). Let $C=\exists(\varnothing \hookrightarrow P, a c)$ and $C^{\prime}=\exists\left(\varnothing \hookrightarrow P^{\prime}, a c^{\prime}\right)$ with composed total negative application conditions $a c=\wedge_{i \in I} \neg \exists\left(P \hookrightarrow^{x_{i}} X_{i}\right)$ and $a c^{\prime}=$ $\wedge_{j \in J} \neg \exists\left(P^{\prime} \hookrightarrow^{x_{j}^{\prime}} X_{j}^{\prime}\right)$ for index sets $I, J$ be two patterns. Then $C^{\prime} \vDash C$, if the following conditions are fulfilled:

1. There exists a monomorphism $m: P \hookrightarrow P^{\prime}$ such that:

2. With Shift $\left(m, \neg \exists x_{i}\right)=\bigwedge_{k \in K_{i}} \neg \exists\left(P^{\prime} \hookrightarrow x_{i k}^{\prime \prime} X_{i k}^{\prime \prime}\right)$ for a number of corresponding index sets $K_{i}$, for each $x_{i}$ it holds that

$$
\forall k\left(k \in K_{i} \Rightarrow \exists j \exists y\left(y: X_{j}^{\prime} \hookrightarrow X_{i k}^{\prime \prime} \wedge x_{i k}^{\prime \prime}=y \circ x_{j}^{\prime}\right)\right) .
$$

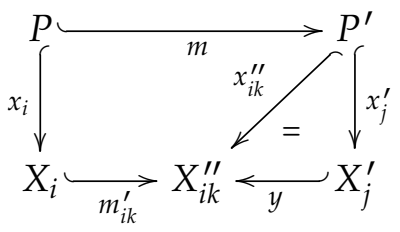

Proof. Assuming that the above conditions hold, we have to show $\forall G\left(G \vDash C^{\prime} \Rightarrow\right.$ $G \vDash C)$.

Consider an arbitrary graph $G$ with $G \vDash C^{\prime}$. By definition of satisfaction, we have $i_{G} \vDash C^{\prime}$, implying the existence of a monomorphism $g^{\prime}: P^{\prime} \leftrightarrow G$ with $g^{\prime} \vDash \mathrm{ac}^{\prime}$. By 
assumption, there is a monomorphism $m: P \hookrightarrow P^{\prime}$. Then there exists a morphism $g$ : $P \rightarrow G$ with $g=g^{\prime} \circ m$. Since $\mathcal{M}$ is closed under composition, $g$ is a monomorphism.

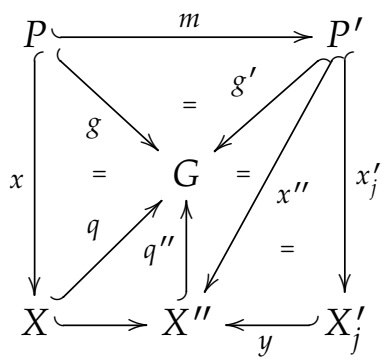

We will show $g \vDash$ ac by contradiction. Suppose $g \notin$ ac, implying the existence of a $x=x_{i}$ for some $i \in I$ and a corresponding monomorphism $q: X \hookrightarrow G$ with $g=q \circ x$, i.e. $g \not \neg \exists x$. For Shift $(m, \neg \exists x)=\wedge_{k \in K} \neg \exists\left(P^{\prime} \hookrightarrow_{k}^{x^{\prime \prime}} X_{k}^{\prime \prime}\right)$ for an index set $K$ depending on $x$, we have $g^{\prime} \vDash \operatorname{Shift}(m, \neg \exists x) \Leftrightarrow g^{\prime} \circ m \vDash \neg \exists\left(x_{i}\right)$ (Lemma 36). Since $g=g^{\prime} \circ m$, we have $g^{\prime} \neq \operatorname{Shift}(m, \neg \exists x)$ and thus $g^{\prime} \vDash \wedge_{k \in K} \neg \exists\left(P^{\prime} \hookrightarrow_{k}^{x_{k}^{\prime \prime}} X_{k}^{\prime \prime}\right)$. This implies the existence of a $x^{\prime \prime}=x_{k}^{\prime \prime}$ for some $k \in K$ with $g^{\prime} \neq \neg \neg\left(P^{\prime} \hookrightarrow^{x^{\prime \prime}} X^{\prime \prime}\right)$. Then there exists a monomorphism $q^{\prime \prime}: X^{\prime \prime} \hookrightarrow G$ with $g^{\prime}=q^{\prime \prime} \circ x^{\prime \prime}$. By assumption, there exists a monomorphism $y: X_{j}^{\prime} \hookrightarrow X^{\prime \prime}$ with $x^{\prime \prime}=y \circ x_{j}^{\prime}$ for some $j \in J$. This implies the existence of a monomorphism $q^{\prime}: X_{j}^{\prime} \leftrightarrow G$ with $q^{\prime}=q^{\prime \prime} \circ y$ and thus $q^{\prime} \circ x_{j}^{\prime}=q^{\prime \prime} \circ y \circ x_{j}^{\prime}=q^{\prime \prime} \circ x^{\prime \prime}=g^{\prime}$. Thus, $g^{\prime} \not \neg \exists x_{j}^{\prime}$ for the specific $j \in J$ and therefore $g^{\prime} \vDash \mathrm{ac}^{\prime}$. This is a contradiction, thus we have $g \vDash$ ac. With $g: P \hookrightarrow G$ and $g \vDash$ ac, we get $G \vDash C$, concluding the proof.

Theorem 41 describes a case for implication of patterns where both pattern contain only composed total negative application conditions. It can be seen that the creation of composed total negative application conditions from composed partial negative application conditions requires a high effort and that the computational effort for checking implication is also usually higher than for the partial case (cf. Theorem 16 since all negative application conditions from the implied pattern have to be shifted to the implying pattern.

Finally, the following lemma describes the translation of composed total negative application conditions defined over a left rule side (as part of a graph rule) to conditions over a source pattern.

Lemma 42 (transformation of left application conditions to source patterns). Let $s c r^{\prime}=\exists\left(s_{p}: L \hookrightarrow S, a c_{S}^{\prime}\right)=L(b$, tar $)$ be a source pattern constructed from a target pattern tar and a rule $b=\left\langle(L \hookleftarrow K \hookrightarrow R), a c_{L}\right.$, true $\rangle$. Let $a c_{S}^{\prime}$ further be a composed partial negative application condition. Then $s c r=\exists\left(s_{p}: L \hookrightarrow S, a c_{S}\right)$ with $a c_{S}=a c_{S}^{\prime} \wedge \operatorname{Shift}\left(s, a c_{L}\right)$ is a source pattern over $L$ and we have: 
1. For each graph $G$ and each monomorphism $m: L \hookrightarrow G:\left(m \vDash s r c^{\prime} \wedge m \vDash a c_{L}\right) \Leftrightarrow m \vDash$ src.

2. For each each direct graph transformation $G \Rightarrow_{b, m, m^{\prime}} H$ we have: $m \vDash s r c \Leftrightarrow m^{\prime} \vDash$ tar.

Proof. Since $\mathrm{ac}_{L}$ is a composed negative appplication condition, according to Lemma $38 \operatorname{Shift}\left(s, \mathrm{ac}_{L}\right)$ is a composed negative application condition. By definition of source patterns, $\mathrm{ac}_{S}^{\prime}$ is a composed negative application condition. Hence, $\mathrm{ac}_{S}^{\prime} \wedge \operatorname{Shift}\left(s, \mathrm{ac}_{L}\right)$ is a composed negative application condition, which makes $\operatorname{src}=\exists(s: L \hookrightarrow$ $\left.S, \operatorname{ac}_{S}^{\prime} \wedge \operatorname{Shift}\left(S, \mathrm{ac}_{L}\right)\right)$ a source pattern.

For the first property, we transform the left side of the equivalence as follows.

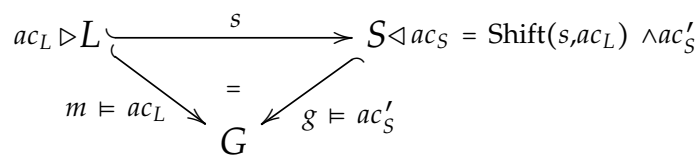

$$
\begin{aligned}
& m \vDash \operatorname{src}^{\prime} \\
& \Longleftrightarrow m \vDash \exists\left(s: L \hookrightarrow S, \mathrm{ac}_{S}^{\prime}\right) \\
& \wedge m \vDash \mathrm{ac}_{L} \\
& \Longleftrightarrow \exists g\left(g: S \hookrightarrow G \wedge m=g \circ S \wedge g \vDash \mathrm{ac}_{S}^{\prime}\right) \\
& \wedge m \vDash \mathrm{ac}_{L} \\
& \Longleftrightarrow \exists g\left(g: S \hookrightarrow G \wedge m=g \circ S \wedge g \vDash \mathrm{ac}_{S}^{\prime}\right. \\
& \wedge m \vDash \mathrm{ac}_{L} \\
& \Longleftrightarrow \exists g\left(g: S \hookrightarrow G \wedge m=g \circ S \wedge g \vDash\left(\mathrm{ac}_{S}^{\prime}\right.\right. \\
& \left.\wedge g \vDash \operatorname{Shift}\left(s, \mathrm{ac}_{L}\right)\right) \quad \text { (Shift-lemma) } \\
& \Longleftrightarrow \exists g\left(g: S \hookrightarrow G \wedge m=g \circ s \wedge g \vDash \mathrm{ac}_{S}\right) \\
& \Longleftrightarrow m \vDash \mathrm{src} \\
& \left.\left.\wedge \operatorname{Shift}\left(s, \mathrm{ac}_{L}\right)\right)\right)
\end{aligned}
$$

This shows the first property.

By the L-lemma, for each direct graph transformation $G \Rightarrow_{b, m, m^{\prime}} H$ we have $m \vDash \operatorname{src}^{\prime} \Leftrightarrow m^{\prime} \vDash$ tar. From $G \Rightarrow_{b, m, m^{\prime}} H$ we get $m \vDash \operatorname{ac}_{L}$. With $\left(m \vDash \operatorname{ac}_{L} \wedge m \vDash\right.$ $\left.\mathrm{src}^{\prime}\right) \Leftrightarrow m \vDash$ src from the first property, we have $m \vDash \operatorname{src} \Leftrightarrow m^{\prime} \vDash$ tar, concluding the proof.

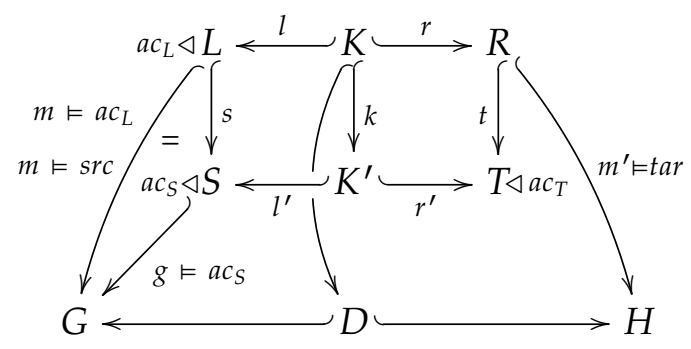




\section{Aktuelle Technische Berichte des Hasso-Plattner-Instituts}

Band ISBN Titel

978-3-86956-334-3 Parts without a whole? : The current state of Design Thinking practice in organizations

Modeling collaborations in selfadaptive systems of systems : terms, characteristics, requirements and scenarios

978-3-86956-324-4 Proceedings of the 8th Ph.D. retreat of the HPI research school on service-oriented systems engineering

Control : Entwurf und Implementierung einer Software zur Optimierung heterogener Energiesysteme in Mehrfamilienhäusern standard documents at Carmeq $\mathrm{GmbH}$ Cloud Symposium "Operating the Cloud" 2014
Development of AUTOSAR

\section{Autoren / Redaktion}

Jan Schmiedgen, Holger Rhinow, Eva Köppen, Christoph Meinel

Sebastian Wätzoldt, Holger Giese

Christoph Meinel, Hasso Plattner, Jürgen Döllner, Mathias Weske, Andreas Polze, Robert Hirschfeld, Felix Naumann, Holger Giese, Patrick Baudisch

Sascha Bosse, Esam Mohamed, Frank Feinbube, Hendrik Müller (Hrsg.)

Eva-Maria Herbst, Fabian Maschler, Fabio Niephaus, Max Reimann, Julia Steier, Tim Felgentreff, Jens Lincke, Marcel Taeumel, Carsten Witt, Robert Hirschfeld

Regina Hebig, Holger Giese, Kimon Batoulis, Philipp Langer, Armin Zamani Farahani, Gary Yao, Mychajlo Wolowyk

Andreas Meyer, Mathias Weske process models and synchronized object life cycles Projects

Uwe Hentschel, Daniel Richter, Andreas Polze

openHPI: 哈索・普拉特纳研究院的 MOOC（大规模公开在线课）计划

Christoph Meinel, Christian Willems

Christian Neuhaus, Andreas Polze (Hrsg.)

Luise Pufahl, Andreas Meyer, Mathias Weske 

ISBN 978-3-86956-333-6

ISSN 1613-5652 